2015-04-30

\title{
A Targeted Needs Assessment for a Transitional "Boot Camp" Curriculum for Pediatric Surgery Residents
}

\author{
Blackmore, Christopher
}

Blackmore, C. (2015). A Targeted Needs Assessment for a Transitional "Boot Camp"

Curriculum for Pediatric Surgery Residents (Unpublished master's thesis). University of Calgary, Calgary, AB. doi:10.11575/PRISM/25053

http://hdl.handle.net/11023/2196

Downloaded from PRISM Repository, University of Calgary 


\title{
UNIVERSITY OF CALGARY
}

A Targeted Needs Assessment for a Transitional "Boot Camp" Curriculum for Pediatric Surgery Residents

by

Christopher Blackmore

\begin{abstract}
A THESIS
SUBMITTED TO THE FACULTY OF GRADUATE STUDIES

IN PARTIAL FULFILMENT OF THE REQUIREMENTS FOR THE

DEGREE OF MASTER OF SCIENCE
\end{abstract}

GRADUATE PROGRAM IN COMMUNITY HEALTH SCIENCES

CALGARY, ALBERTA

APRIL, 2015

(C) Christopher Blackmore 2015 


\begin{abstract}
Transition periods in medical education are associated with increased risk for learners and patients. In pediatric surgery, the transition to specialty is especially difficult as learners adjust to new patient populations.
\end{abstract}

In this study we performed a targeted needs assessment to determine the ideal content and format of a pediatric surgery boot camp to aid in the transition to residency. The survey was distributed to pediatric surgery residents and staff across North America.

No significant differences were identified between staff and residents in survey responses. The top 5 topics identified for inclusion in a boot camp were: 1) Fluid and electrolyte management, 2) Appendicitis, 3) Pediatric Hernias, 4) Nutrition and 5) Pain Management. The preferred duration for a boot camp was 3-4 days using a variety of educational methods including elearning, simulation and small group teaching.

Based on results of the needs assessment survey, a novel pediatric surgery boot camp curriculum can be developed. 


\section{Acknowledgements}

Since the moment I decided to pursue a post-graduate degree, I have had immense support from all of those around me. First and foremost, I would like to thank my supervisor, Dr. Elizabeth Oddone-Paolucci. It was her constant support and expert guidance that helped me to bring this project from a dream to reality. I was fortunate to have you as a supervisor.

I would also like to extend many thanks to Dr. Steve Lopushinsky who has acted not only as a committee member, but also as a mentor. You have shown me what it means to be an academic surgeon, and I hope to be able to mirror your well balanced approach to life and medicine as I develop my career as a surgeon.

A huge thank you also goes out to the remaining member of my committee, Dr. Jocelyn Lockyer, for her expert guidance during my curricular design project. Calgary is lucky to have so many experts in medical education involved in the Community Health Sciences program.

I would also like to thank my external examiner, Dr. Mark Walton, for offering his expertise as an educator and pediatric surgeon. I greatly appreciate you volunteering your time to review my manuscript and take part in the examination. I look forward to meeting you again in a less stressful environment!

I owe a thank you to the Department of Surgery for providing financial support for the project through the Calgary Surgical Research and Development Fund. And last but not least, a huge thank you to my family, especially my wife who supported me during my year off, both emotionally and financially, and my dogs who sat on the couch many hours staring at me as I wrote this thesis. 


\section{Dedication}

I would like to dedicate this thesis to my parents, Peter and Cristina Blackmore. Since the moment I chose to embark on the long path to becoming a doctor, to the moment I chose to prolong this path with a post-graduate degree and sub-specialty training, they have been there to support me. Thank you for always being there for me and never letting me lose sight of my dreams. 


\section{Table of Contents}

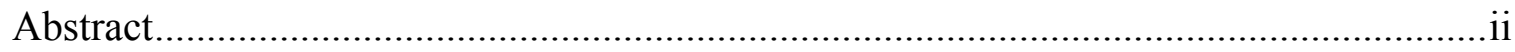

Acknowledgements ................................................................................................ii

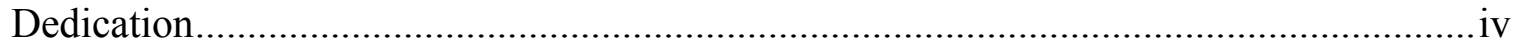

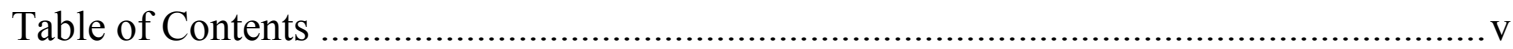

List of Tables ..............................................................................................................

List of Symbols, Abbreviations and Nomenclature …………..........................................viii

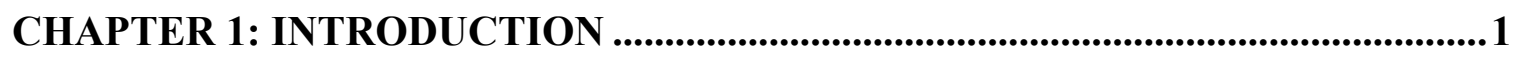

1.1 Overview of Research Project.......................................... 2

1.2 Aim ............................................................... 2

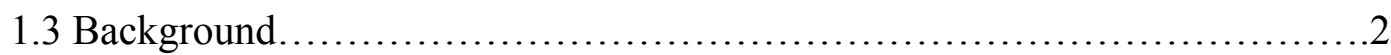

1.3.1 Transitions in Medical Education................................2

1.3.2 Educational Models During Critical Transition Periods................ 4

1.3.3 Boot Camps as a Tool During Transition Periods......................6

1.4 Knowledge Gaps and Significance......................................

1.5 Objectives............................................................ 8

CHAPTER 2: A TARGETED NEEDS ASSESSMENT FOR A TRANSITIONAL "BOOT CAMP" CURRICULUM FOR PEDIATRIC SURGERY RESIDENTS ......9

2.1 Introduction....................................................... 10

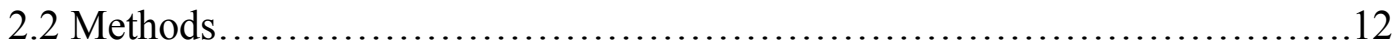

2.2.1 Participants..................................................... 12

2.2.2 Targeted Needs Assessment Survey Development..................12

2.2.3 Data Analysis.................................................. 14

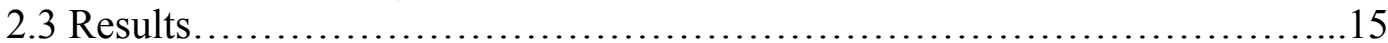

2.3.1 Participants..................................................... 15

2.3.2 Target Needs Assessment Survey: Boot Camp Content.............15

2.3.3 Rank Order Method Comparison................................... 15

2.3.4 Targeted Needs Assessment: Boot Camp Format..................16

2.4 Discussion............................................................ 17

2.5 Acknowledgements............................................. 20

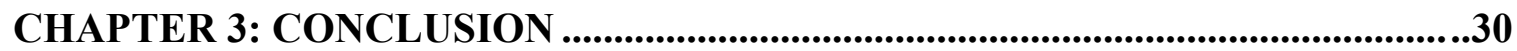

3.1 Summary of Main Study Findings................................... 31

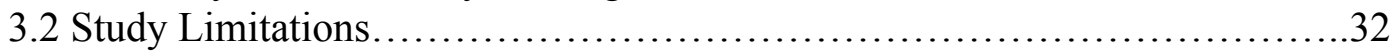

3.3 Directions of Future Research......................................... 33

3.4 Patient and Learner Implications..................................... 34

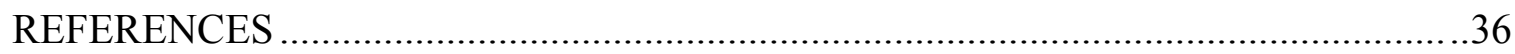

APPENDIX A: BOOT CAMP SYSTEMATIC REVIEW AND METANALYSIS

MANUSCRIPT WITH PERMISION FOR USE FROM JOURNAL ......................44 
APPENDIX B: NEEDS ASSESSMENT SURVEY MANUSCRIPT WITH PERMISSION

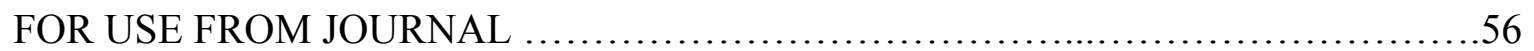

APPENDIX C: NEEDS ASSESSMENT SURVEY....................................64

APPENDIX D: EXAMPLE OF LIKERT SCALE USED IN NEEDS ASSESSMENT

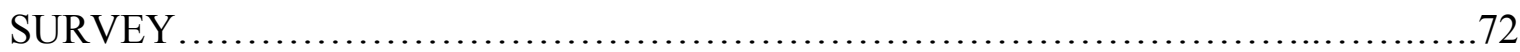

APPENDIX E: SURVEY CONSENT FORM.......................................73

APPENDX F: CONTRIBUTION OF AUTHORS .......................................76 


\section{List of Tables}

Table 1: Pediatric Surgical Diagnoses Frequency and Importance Ratings by Residents and Staff $.21-22$

Table 2: Pediatric Clinical and Surgical Skills Frequency and Importance Ratings by Residents and Staff

Table 3: Pediatric Physiology Topic Frequency and Importance Ratings by Residents and Staff

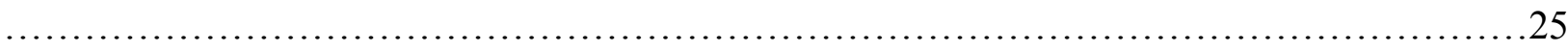

Table 4: Rank Order Method Comparison Using Combined Data for Residents and Staff....26-29 


\section{List of Symbols, Abbreviations and Nomenclature}

Symbol
SBME
SBML
PGME
RCPSC
ACGME
NAS
CAPS
MANOVA

Definition

Simulation Based Medical Education

Simulation Based Mastery Learning

Post Graduate Medical Education

Royal College of Physicians and Surgeons of Canada Accreditation Council for Graduate Medical Education Next Accreditation System

Canadian Association of Pediatric Surgeons

Multivariate Analysis of Variance 
CHAPTER 1: INTRODUCTION 


\subsection{Overview of Research Project}

The following thesis research involved targeting key stakeholders through a needs assessment 2 aimed at evaluating the perceived need for a pediatric surgery boot camp. As a result, this document includes a: (i) description of the literature as it pertains to medical education boot camps and their use during critical transition periods; (ii) report on the development and subsequent results of the targeted needs assessment; and (iii) discussion of the implications of this work, along with recommendations for future steps.

\subsection{Aim}

The primary aim of the thesis project was to identify the perceived needs of a pediatric surgery boot camp, particularly with regards to the critical curricular content, as well as the preferred format and educational methods. The secondary aim was to report on the process undertaken in a clear and transparent manner, in order to promote reproducibility of future curricular design projects.

\subsection{Background}

\subsubsection{Transitions in Medical Education}

Pediatric Surgery is unique amongst surgical and medical specialties in that trainees obtain their initial specialization in adult surgery before embarking upon the world of pediatrics. In North America, this involves five years of adult general surgery training followed by two years of pediatric surgery subspecialty training. At the start of pediatric surgery residency, trainees have varying degrees of exposure to the pediatric population along with varied levels of comfort addressing pediatric specific problems. Furthermore, this transition to subspecialty training comes with a sense of increased personal responsibility towards patients.[1] Not 
surprisingly, during informal discussions with pediatric surgery residents and staff, the initial transition into the subspecialty training has been described as a stressful experience.

The concept of stressful career transitions in medical and surgical training is not a new one. For instance, the transition from pre-clerkship to clerkship training has been found to be stressful for medical students due to the perceived increased workload.[2,3] Multiple studies have cited the transition from medical student to surgical resident as a particularly difficult transition for learners, often due to the variability in training with regards to surgical skills during clerkship, as well as the increased number of clinical responsibilities that come with being a resident.[4-7] Similarly, the transition from residency to fellowship or subspecialty training has been cited as a time of increased stress given the complexity of patients and the increased expectation of academic performance without failure.[1,8] These transition periods have also been associated with increased rates of psychiatric morbidity and burnout amongst trainees. [9]

Transition periods in medical training not only affect the wellbeing of the learner, but there is potential risk for the patient as well. Investigators have spent a significant amount of time and resources examining morbidity and mortality rates during transition periods. These studies have identified mixed results. Some have found little evidence for a "July Phenomenon", with similar rates of mortality and serious adverse events during the first part of the academic year and subsequent months.[10-13] Other studies have found increased rates of adverse events with no significant increase in mortality.[14,15] On the other end of the spectrum, some studies have found evidence of both increased morbidity and mortality amongst patients admitted to teaching hospitals in July.[16,17]

In light of the mixed results from prior studies, a systematic review was performed by Young et al. [18] to assess the effects of transition periods on patient outcomes. In total 39 
studies were included in their analysis, with 27 reporting on mortality, 19 reporting on efficiency, 23 reporting on morbidity, and 6 reporting rates of medical errors. Of the included articles, 13 were identified to be of higher quality. Amongst the higher quality studies, statistically significant increased rates of mortality and decreased efficiency were identified during transition periods (i.e. July). The poorer quality studies largely reported on morbidity and medical errors with inconsistent results.[18] Given the medico-legal and ethical concerns associated with increased morbidity or mortality amongst patients, in addition to the concerns regarding health care costs related to increased rates of complications and overall decreased efficiency during transition periods, there has been a strong push towards new training methods to teach surgical trainees to mitigate these effects.[19,20]

\subsubsection{Educational Models During Critical Transition Periods}

Traditionally, training for most surgical specialties was in the form of a master-apprentice relationship in which trainees observed their staff in practice. As the trainee became more familiar with surgical skills they would begin to perform more intricate tasks, at first under guidance and then independently.[19,21] In this model of training, there was little emphasis on what should be taught, who should be taught, or how it should be taught.[22]

The apprentice model of surgical education prevailed until the turn of the $20^{\text {th }}$ century when efforts by Dr. William S. Halsted, Dr. William Osler, and recommendations by Abraham Flexner led to the development of the Halstedian model of surgical training.[22,23] The Halstedian model brought with it a more formalized and structured education, with higher admission requirements, where residents were expected to acquire an understanding of the disease processes.[22] In this model, residents were presented with repetitive opportunities to 
take care of surgical patients under supervision from a skilled surgeon, and acquired surgical skills in a graded fashion.[22]

Building upon the Halstedian model, Ericsson focused on the process through which residents transformed from unskilled trainees to master surgeons.[24] In his studies, Ericsson examined experts in various fields, including athletics, music and chess. Through his research Ericsson found little correlation between the length of training and an individuals observed level of skill.[24] Rather, according to Ericsson's model of expert performance, the key to mastery of surgical skills is an active engagement in deliberate practice with opportunities for immediate feedback and repeated performance to refine behaviours.[24] This concept is key in the mastery learning model that began to prevail later in the $20^{\text {th }}$ century leading up to current day. In this model, the salient features include deliberate practice along with a focus on the development of clear learning objectives, baseline and diagnostic assessment, fixed minimum passing standards, and formative assessment with specific feedback.[25] In the mastery model, advancement to the next level, depends on the attainment of the minimum standard as opposed to time spent training.[25]

With the implementation of work hour restrictions, the focus of surgical education shifted once again. As time spent directly interacting with patients on the ward or in the operating room became more scarce, simulation based medical education (SBME) began to emerge as a new method of training surgeons. SBME allows trainees to practice clinical skills in controlled environments where formative feedback is available.[25] Compared to traditional methods of education, SBME has proved a highly effective method in medical education.[26] Combined with the mastery learning model, simulation based mastery learning (SBML) produces not only 
improvements in the simulation laboratory, but also safer patient care practices, better patient outcomes, and potential cost savings.[26-30]

Using Ericsson's model and SBML as a guide, many programs across North America are developing surgical "boot camps" designed to ease learners into their new roles and to start them on the path to becoming experts in their respective fields. In the Competence by Design initiative set forth by the Royal College of Physicians and Surgeons of Canada, the need for structured support for learners during transition periods is highlighted, with the main drivers for change being patient safety, stress upon the learners, and productivity.[20] While boot camps have been described for various surgical specialties, no such training camp exists in North America for pediatric surgery.

\subsubsection{Boot Camps as a Tool During Transition Periods}

In order to determine the overall effectiveness of the boot camp approach to easing stressful career transitions, we performed a meta-analytic review of surgical boot camps (Appendix A).[31] Based on a literature review, alternative wording for "boot camp" was identified to include "capstone" course, "crash" course, "transition" course and "rookie" camp. Our search was performed using MEDLINE, CINAHL, PSYCinfo, EMBASE and ERIC with "boot camp" and its alternate wordings as the focus of our search.

The inclusion criteria for the meta-analysis comprised those studies that reported on medical or surgical boot camps, involved learners entering new clinical roles in North American programs, and reported empirical data on the effectiveness of boot camps to improve clinical skills, knowledge and/or confidence. In total, 15 articles met all inclusion criteria.

In identifying the articles to include in our meta-analysis, we encountered a variety of formats for educational boot camps. Although differences existed between individual boot 
camps, certain commonalities were present: all studies described boot camps as courses aimed to prepare or orient learners undergoing clinical transitions periods, boot camps were structured as short and focused courses, and a wide variety of educational methods were applied in each camp with a focus on simulation. In addition, in keeping with Ericsson's theory of expert performance [24], most boot camps emphasised the provision of immediate and/or formative feedback to participants.

From the descriptions of boot camps identified in our review, we developed an allencompassing definition of a "boot camp" as follows:

A boot camp is a focused course designed to enhance learning, orientation and preparation for learners entering a new clinical role. This is achieved through the use of multiple educational methods with a focus on deliberate practice with formative feedback.

Amongst the 15 articles that met inclusion criteria, three broad groups of outcomes were measured before and after the boot camp interventions: (1) knowledge, (2) confidence, and (3) skills. Statistical analyses were conducted for each of the three main outcome measures of knowledge, confidence and skills using Cohen's d for effect size and the Random Effects Model.[32] The meta-analytic combined effects showed that participation in surgical boot camps significantly improved the learner's knowledge $(\mathrm{d}=2.08,95 \%$ CI: $1.20-2.96, \mathrm{p}<.001)$, confidence $(\mathrm{d}=1.89,95 \%$ CI: 1.63-2.15, $\mathrm{p}<.001)$, and skills $(\mathrm{d}=1.78,95 \%$ CI: 1.33-2.22, $\mathrm{p}<$ $.001)$.

\subsection{Knowledge Gaps and Significance}

The results of our meta-analysis revealed that surgical boot camps can be effective at improving learners' knowledge, confidence and skills in a variety of domains related to surgical 
specialties. The studies included in the meta-analysis were all implemented during the transition from the role of medical student to surgical resident, taking place either at the end of medical school or start of residency. Despite the complexity of the transition from practicing surgery on an adult population to a pediatric population with unique physiology and novel disease processes, to our knowledge, no study has focused on developing a boot camp to aid pediatric surgery residents through the transition to subspecialty training.

\subsection{Objectives}

The main objective of the current study, therefore, was to use what we learned in the empirical integration of published data (i.e., meta-analysis) on surgical boot camps to conduct a targeted needs assessment for a pediatric surgery boot camp, eventually leading to the development of a new competence based pediatric surgery boot camp curriculum. The target audience for this boot camp would be residents transitioning into the role of pediatric surgery after completing five years of adult general surgery training. Given the overlap in many CanMEDS competencies between adult and pediatric training, including the collaborator, scholar and manager competencies, the primary focus of our boot camp would be to address the areas participants are least familiar with, particularly the novel medical expert competencies required for a pediatric surgery resident.

The individual objectives for the study are as follows: (1) identify differences in the perceived needs for a pediatric surgery boot camp amongst stakeholders; (2) assess empirical ranking methods to inform the development of the future curriculum; (3) identify which pediatric surgical diagnoses, skills, and physiological topics should be included as curricular content in a pediatric surgery boot camp for new residents; and (4) determine the preferred boot camp duration, structure and educational methods to be employed. 
CHAPTER 2: A TARGETED NEEDS ASSESSMENT FOR A TRANSITIONAL "BOOT CAMP" CURRICULUM FOR PEDIATRIC SURGERY RESIDENTS 


\subsection{Introduction}

Medical education in North America consists of well-defined stages connected by critical transition periods as one progresses from medical school to clinical practice. Focusing on PostGraduate Medical Education (PGME), multiple studies have cited the transition from medical school to surgical residency as being stressful due to the variability in surgical skills exposure during clerkship as well as the increased number of clinical responsibilities.[4-7] These transition periods have also been associated with higher rates of psychiatric morbidity and burnout amongst trainees.[9]

Of concern to medical educators and the community at large is that transition periods may introduce potential risk for patients. The "July Phenomenon" is a well-studied effect predicting increased patient morbidity and mortality at teaching hospitals at the start of the academic year; a time that parallels most major transitions in medical training. In support of this, recent studies have shown that there is an increased risk of errors accounting for preventable complications during July.[10,11]

From a pediatric surgery perspective, the Canadian Pediatric Adverse Events Study has identified that more children experience adverse events in academic centres compared to community centres.[33] This discrepancy was most pronounced in surgical patients, where those treated at academic centres $(37.2 \%$ of patients) experienced higher rates of adverse events compared to community centres (21.5\% of patients).[33] Although confounding factors exist, including the increased complexity and higher acuity of patients encountered at academic centres, this study clearly highlights a need for reduction of adverse events, especially among pediatric surgical patients at academic centres. 
Given the above concerns, there has been a strong push towards new methods of teaching and evaluation in PGME.[20] Programs are beginning to adopt competency-based frameworks to ensure that individual learner's skills match their progress through the continuum of medical education. In Canada, this is exemplified by the Royal College of Physicians and Surgeons of Canada (RCPSC) Competence by Design initiative.[34] In the United States, the Accreditation Council for Graduate Medical Education (ACGME) is implementing the Next Accreditation System (NAS) based on competency milestones.[35] In addition, many surgical programs have adopted "boot camps" to train residents during the critical transition to residency.[19,36-38]

Pediatric surgery is unique in that trainees obtain their initial specialization in adult general surgery before transitioning to pediatrics. At the start, trainees have varying degrees of exposure to pediatrics and varying comfort levels in addressing pediatric issues. During informal discussions with pediatric surgery residents and staff, the transition into pediatric surgery has been described as particularly difficult. As a result, it was timely to investigate the perceptions of residents and staff regarding the need to develop an educational intervention for new pediatric surgery residents.

The purpose of this study was to conduct a targeted needs assessment of key stakeholders (i.e., residents and staff) in order to: (1) identify differences in the perceived needs for a pediatric surgery boot camp amongst stakeholders; (2) assess empirical ranking methods to inform the development of the future curriculum; (3) identify which pediatric surgical diagnoses, skills, and physiological topics should be included as curricular content in a pediatric surgery boot camp for new residents; and (4) determine the preferred boot camp duration, structure and educational methods to be employed. To the best of our knowledge, this study was the first of its kind to 
gather the information necessary to inform the development of a pediatric surgery boot camp curriculum.

\subsection{Methods}

\subsubsection{Participants}

Participants comprised the entire population of the Canadian Association of Pediatric Surgeons (CAPS; $\mathrm{n}=172$ ). Additionally, Canadian and American trainees were targeted via their program directors who were asked to forward the needs assessment survey to their current residents. Although exact numbers could not be determined, the survey was estimated to have been distributed to approximately 200 participants.

\subsubsection{Targeted Needs Assessment Survey Development}

In consultation with pediatric surgery staff, residents and a curricular design expert (J.L) at the University of Calgary, a targeted needs assessment survey was developed. The survey consisted of two parts: (1) boot camp content, and (2) boot camp format (Appendix C). For the content section, three overarching domains within medical expert competencies were identified: (1) pediatric surgical diagnoses; (2) pediatric clinical and surgical skills; and (3) pediatric physiology. To ensure content validity, each of the pediatric surgical diagnoses $(n=30)$, skills $(\mathrm{n}=20)$, and physiology topics $(\mathrm{n}=11)$ included in the survey were derived directly from the RCPSC Objectives in Training for Pediatric Surgery medical expert competencies.[39] These objectives closely mirror those outlined by the ACGME Pediatric Surgery Milestone Project.[40] Additional CanMEDS competencies, including scholar, patient advocate and manager, were left out of the content section of the survey as it was felt that the targeted audience for a boot camp would have had the opportunity to master these competencies during 
their residency training in adult general surgery. As such, the novel medical expert competencies encountered during pediatric surgery residency were the primary focus.

Participants were asked to rate each of the pediatric surgical and diagnoses, skills, and physiology topics on the level of "importance" to new pediatric surgery residents during their transition to training, and the "frequency" with which these items were encountered by pediatric surgeons or residents. This was done using a 5-point Likert scale (Appendix D). For importance, the scale ranged from "1" referring to "Not at all Important" to a score of "5" referring to "Very Important." For frequency, the scale ranged from "1" referring to "Less than Once per Year" to "5" referring to "More than Once per Week."

Our rating system was adapted from a similar study performed to create a rank order list of topics for inclusion in an internal medicine examination blueprint.[41] The "importance" score was designed to incorporate topics deemed critical for new trainees based on their level of urgency and room for error. The "frequency" score aimed to capture topics encountered most often by a new trainee. By combining these factors as described below, we aimed to create a rank order list of topics deemed most critical for a pediatric surgical boot camp. This list would be used to develop goals, objectives and ultimately a pediatric surgery transitional "boot camp" curricular outline.

The boot camp format section of the survey involved multiple choice, short answer, and open-ended questions to determine the preferred format of the boot camp. Specifically, questions regarding the perceived ideal duration, instructional methods, and structure were posed. Given that the content section focused primarily on medical expert competencies, in the open-ended section of the survey participants were also asked to describe other competencies or additional content areas they felt should be included in a pediatric surgery boot camp. 
After development of the preliminary survey, it was pilot tested by a pediatric surgeon and pediatric surgery resident at the University of Calgary. Subsequent iterations of the survey were piloted in a similar manner until it was felt that face validity was achieved. The survey was created using SurveyMonkey (SurveyMonkey Inc., Palo Alto, California, USA) and distributed via an emailed electronic link, with one reminder email that was delivered at approximately two weeks. Data were collected anonymously.

\subsubsection{Data Analysis}

All statistical analyses were performed using SPSS version 22 (SPSS Inc., Chicago, Illinois, U.S.A). For the content part of the survey, the mean scores and standard deviations were calculated for importance and frequency ratings for all staff and resident respondents. For each domain (pediatric surgical diagnoses, skills and physiology), multivariate analysis of variance (MANOVA) was applied comparing staff and residents to identify any differences in how the key stakeholders perceived topics on the level of importance or frequency.

To determine which content should be included in a boot camp curriculum, we applied methodology described by Smith and Beran [42] for the development of rank order lists for clinical presentations. Given our smaller sample size, we were able to test four of the five methods for combining ratings of frequency and importance. The Rasch model was excluded from analysis given its requirement for large sample sizes.[42] The first method involved ranking based solely on "frequency" which determines how often the topic is encountered in practice. The second method involved ranking on "importance" ratings. The third method, the multiplicative model, involved multiplying the "frequency" and "importance" ratings to obtain a composite score, which provided equal weighting to both ratings. Lastly, the three-step model is 
a form of assigning weights on both frequency and severity. In this model, items ranked highly on both scales received higher weighting than items ranked highly on a single scale.[42]

For the boot camp format section of the survey, answers were tabulated using frequencies. Open-ended questions were transcribed and themed. The University of Calgary Conjoint Health Research Ethics Board approved this study.

\subsection{Results}

\subsubsection{Participants}

In total 12 residents and 23 pediatric surgery staff completed our survey, representing an estimated response rate of $18 \%(35 / 200)$. Overall, $57 \%(n=20)$ of survey respondents were male, and $57 \%(n=20)$ had completed or were registered in training programs in Canada, $40 \%(n=14)$ had completed or were registered in programs within the United States, and 3\% $(n=1)$ had completed training outside of North America.

\subsubsection{Targeted Needs Assessment Survey: Boot Camp Content}

Mean ratings were tabulated for frequency and importance for residents and staff for topics under each domain: pediatric surgical diagnoses (Table 1), pediatric surgical skills (Table 2), and pediatric physiology (Table 3). The MANOVAs comparing resident and staff scores for importance, as well as frequency, revealed no significant differences between stakeholders (Tables 1-3).

\subsubsection{Rank Order Method Comparison}

For the topics within each domain (pediatric surgical diagnoses, skills and physiology), the four ranking methods were applied and compared with Spearman's correlations $(\rho)$ in order to determine consistency amongst methods (Table 4). All four rankings methods were significantly correlated to one another. The frequency and importance methods had the lowest 
correlation $(\rho=0.59, p<.01)$, while the three-step model and multiplicative model were the most highly correlated methods $(\rho=0.99, p<.01)$.

\subsubsection{Targeted Needs Assessment: Boot Camp Format}

Of the domains evaluated by our survey, participants ranked pediatric surgical diagnoses as most important, pediatric physiology as the second most important, and pediatric surgical skills as least important to focus on in a pediatric surgery boot camp.

The favoured duration of the boot camp was three to four days $(n=12 ; 34 \%)$, followed by five to six days $(n=8 ; 23 \%)$, seven days or longer $(n=7 ; 20 \%)$, and lastly one to two days $(n=5$; 14\%). A small group of survey respondents were undecided $(n=3 ; 9 \%)$.

To evaluate preferred educational methods, participants were asked to choose from a list one or more methods that were best suited to teach each domain. For pediatric surgical diagnoses, the top three educational methods identified in the survey were problem based learning $(n=29 ; 83 \%)$, e-learning $(n=26 ; 74 \%)$ and small group teaching $(n=24 ; 69 \%)$. For pediatric surgical skills, the top three methods were high fidelity simulation $(n=26 ; 74 \%)$, animal labs $(n=23 ; 66 \%)$ and low fidelity simulation $(n=20,57 \%)$. Lastly, for pediatric physiology, the top three educational methods were problem based learning $(n=26 ; 74 \%)$, e-Learning $(n=25$; $71 \%)$ and didactic lectures $(n=24 ; 69 \%)$.

In the open-ended section of the survey, participants were asked if there were additional competencies that should be included in a pediatric surgery boot camp. The most common theme identified by participants $(n=10 ; 29 \%)$ was to include a component on communication. This included communication with patients, difficult or anxious parents, surgical staff and pediatric colleagues. Other areas mentioned included pediatric drug dosing, recognizing pediatric abuse, and patient advocacy. 


\subsection{Discussion}

This study consisted of developing and distributing a targeted needs assessment survey to key stakeholders in the field of pediatric surgery. Participants were surveyed regarding the content and format for a pediatric surgery boot camp. No significant differences were identified in survey responses between residents and staff indicating that all stakeholders, regardless of level of training, had similar perceptions as to which topics were critical for new trainees. Given these findings, the data were combined for residents and staff on all further analyses, thereby producing a total of 35 survey respondents.

In addition, four separate methods were evaluated for ranking topics to guide curricular content. While there were statistically significant correlations between all methods, the multiplicative and three-step models showed the most precise rankings, demonstrating a high correlation between both frequency and importance. These models demonstrated that participants tended to rank topics more consistently when both importance and frequency were taken into account, as opposed to independent consideration. In the analysis by Smith and Beran,[42] the Rasch model was concluded to be the ideal method, although it was found to be highly correlated $(0.94, p<.001)$ with the multiplicative model. Given Rasch modeling's requisite of large sample sizes and special software for its application, these factors may serve as a deterrent and limit its utility in the setting of curricular design. With this in mind, the multiplicative model was chosen as our preferred method given its simplicity in application compared to the more cumbersome three-step model or the Rasch model.

Applying the multiplicative model to the data from the content section of the survey, we identified the following as the top three ranked pediatric surgical diagnoses: (1) appendicitis, (2) pediatric hernias, and (3) pediatric burns and trauma. The top three pediatric clinical and 
surgical skills were: (1) vascular access, (2) advanced laparoscopy, and (3) advanced trauma life support. Lastly, the top three pediatric physiology topics were: (1) fluid and electrolyte management, (2) nutrition, and (3) pediatric pain management. These lists represent topics felt by stakeholders to be critical for new pediatric surgery residents to be familiar with due to the high likelihood that they will encounter them during their initial transition into the program, as well as the importance for residents to have an understanding of the topic given factors like low room for error or urgency in which it must be dealt with.

The format section of the survey identified that three to four days was the preferred duration for a pediatric surgery boot camp by over a third of participants. However, $20 \%$ of participants chose 7 days or longer as the ideal format, indicating a preference towards a more longitudinal style curricula. Furthermore, participants felt that content was best addressed using a variety of educational methods including problem based learning, small group teaching, elearning, didactic lectures, and simulation. When structuring the curriculum, participants felt that the focus should be primarily on critical pediatric surgical diagnoses and pediatric physiology. Pediatric surgical skills on the other hand were rated as least important. This was mirrored in the rank order list in which 8 of the top 20 ranked topics were related to pediatric surgical diagnoses, 8 were related to pediatric physiology, and only 4 were related to surgical skills (Table 4). These findings are not surprising given the significant differences between adult diagnoses and physiology compared to the novel pediatric diagnoses and physiology new residents face at the start of their training. Surgical and clinical skills on the other hand demonstrate significant overlap between adult and pediatric surgery, likely explaining why these were felt to be less important for new residents. Lastly, in addition to medical expert 
competencies, participants felt that a boot camp would benefit from including other competencies, specifically communication and health care advocacy.

Combining the boot camp format preferences, from a feasibility perspective, a Friday to Sunday weekend course at a central location would allow for pooling of resources between programs across the country with minimal absence from training for residents. Furthermore, learner and educator time and resource commitment could be enhanced by implementing a blended curriculum, involving an online e-learning component followed by a focused course involving didactic lectures, problem based learning, and simulation. Ideally, this type of boot camp course would supplement longitudinal curricula, while focusing on orientation and evaluation during the transition to discipline for new residents.

The biggest limitation to our study was the low number of survey respondents. Given the small number of pediatric surgical specialists across North America, we were limited in the total population. Using an electronic survey for data collection may have further impacted this given our reliance on program directors for survey distribution. Nonetheless, our response rate was found to be comparable to and consistent with other studies using electronic surveys.[43] In spite of these shortcomings, the combination of resident and staff data enabled us to attain reasonable numbers for appropriate statistical analysis. Furthermore, generalizability of results was maintained through surveying residents and staff across North America.

Through our targeted needs assessment, we were able to determine the educational needs for new pediatric surgery residents, as well the desired format through which these needs can be addressed during the transition into residency. Additionally, we have demonstrated an easily reproducible method of developing rank order lists of topics for inclusion in novel curricula. 
Going forward, the data collected from our survey will be used to develop a new competence based pediatric surgery boot camp curriculum.

\subsection{Acknowledgements:}

Thank you to the Department of Surgery, University of Calgary for providing financial support for this project through the Calgary Surgical Research and Development Fund. 
Table 1: Pediatric Surgical Diagnoses Frequency and Importance Ratings by Residents and Staff $(n=35)$

\begin{tabular}{|c|c|c|c|c|}
\hline \multirow[b]{2}{*}{ Pediatric Surgical Diagnoses } & \multicolumn{2}{|l|}{ Frequency Data } & \multicolumn{2}{|c|}{ Importance Data } \\
\hline & $\begin{array}{l}\text { Staff Mean (SD) } \\
n=23\end{array}$ & $\begin{array}{l}\text { Resident Mean (SD) } \\
\mathrm{n}=12\end{array}$ & $\begin{array}{l}\text { Staff Mean (SD) } \\
n=23\end{array}$ & $\begin{array}{l}\text { Resident Mean (SD) } \\
\mathrm{n}=12\end{array}$ \\
\hline Appendicitis & $4.8(0.4)$ & $5.0(0.0)$ & $4.7(0.5)$ & $4.8(0.5)$ \\
\hline Pediatric Hernias & $4.9(0.3)$ & $4.8(0.4)$ & $4.8(0.4)$ & $4.8(0.9)$ \\
\hline Pediatric Burns and Trauma & $4.2(0.9)$ & $4.4(0.9)$ & $4.3(1.0)$ & $4.6(0.5)$ \\
\hline Pyloric Stenosis & $4.0(0.5)$ & $4.0(0.4)$ & $4.7(0.5)$ & $4.8(0.5)$ \\
\hline Necrotizing Enterocolitis & $3.7(0.7)$ & $3.9(0.5)$ & $4.8(0.4)$ & $4.9(0.3)$ \\
\hline Neonatal Bowel Obstruction & $3.6(0.6)$ & $3.8(0.8)$ & $4.8(0.4)$ & $4.8(0.4)$ \\
\hline Short Bowel Syndrome & $3.4(0.7)$ & $4.0(0.6)$ & $4.3(0.8)$ & $4.3(0.8)$ \\
\hline Abdominal Wall Defects & $3.4(0.7)$ & $3.7(0.5)$ & $4.7(0.4)$ & $4.7(0.7)$ \\
\hline Intussusception & $3.6(0.5)$ & $3.3(0.9)$ & $4.7(0.6)$ & $4.4(0.5)$ \\
\hline Solid Malignancies & $3.4(0.6)$ & $3.6(0.5)$ & $4.3(0.8)$ & $4.4(1.2)$ \\
\hline $\begin{array}{l}\text { Esophageal Atresia/Trachea- } \\
\text { Esophageal Fistulas }\end{array}$ & $3.1(0.5)$ & $3.3(0.7)$ & $4.6(0.7)$ & $4.8(0.9)$ \\
\hline $\begin{array}{l}\text { Anorectal Conditions and } \\
\text { Malformations }\end{array}$ & $3.2(0.5)$ & $3.2(0.9)$ & $4.6(0.7)$ & $4.7(1.2)$ \\
\hline $\begin{array}{l}\text { Congenital Diaphragmatic } \\
\text { Hernia }\end{array}$ & $3.0(0.7)$ & $3.3(0.6)$ & $4.5(0.7)$ & $4.8(0.6)$ \\
\hline Hirschsprungs Disease & $3.1(0.5)$ & $3.3(0.8)$ & $4.7(0.5)$ & $4.7(0.9)$ \\
\hline $\begin{array}{l}\text { Congenital and Acquired Lung } \\
\text { Lesions }\end{array}$ & $3.1(0.5)$ & $3.2(0.6)$ & $4.3(0.8)$ & $4.3(1.2)$ \\
\hline Acute Scrotum & $3.0(0.9)$ & $3.0(0.9)$ & $4.4(0.7)$ & $4.4(0.5)$ \\
\hline Inflammatory Bowel Disease & $3.3(0.5)$ & $3.4(0.5)$ & $4.0(0.9)$ & $3.9(0.8)$ \\
\hline
\end{tabular}




\begin{tabular}{|c|c|c|c|c|}
\hline $\begin{array}{l}\text { Gastrointestinal and Airway } \\
\text { Foreign Bodies }\end{array}$ & $3.2(1.0)$ & $2.9(1.4)$ & $3.8(1.1)$ & $4.2(0.9)$ \\
\hline $\begin{array}{l}\text { Congenital/Acquired Neck } \\
\text { Lesions }\end{array}$ & $3.6(0.7)$ & $3.3(0.7)$ & $3.7(1.2)$ & $3.7(1.1)$ \\
\hline Chest Wall Defects & $3.7(0.9)$ & $3.1(0.7)$ & $3.3(1.1)$ & $3.7(1.2)$ \\
\hline Congenital Heart Anomalies & $3.7(1.1)$ & $3.9(1.6)$ & $3.0(1.0)$ & $3.3(1.4)$ \\
\hline $\begin{array}{l}\text { Pediatric Gastrointestinal } \\
\text { Bleeding }\end{array}$ & $3.0(0.8)$ & $3.1(0.8)$ & $3.9(0.9)$ & $3.8(0.8)$ \\
\hline Pediatric Obesity & $3.4(1.3)$ & $3.8(1.2)$ & $2.9(1.1)$ & $3.3(1.0)$ \\
\hline Genitourinary Conditions & $3.5(1.0)$ & $3.1(1.4)$ & $3.3(1.1)$ & $3.3(1.2)$ \\
\hline Hematologic Malignancies & $3.6(0.8)$ & $3.7(0.7)$ & $3.3(0.9)$ & $3.0(1.0)$ \\
\hline $\begin{array}{l}\text { Congenital Hepatobiliary } \\
\text { Abnormalities }\end{array}$ & $2.6(0.7)$ & $2.4(0.9)$ & $4.2(0.8)$ & $4.2(1.2)$ \\
\hline Gynecologic Conditions & $3.0(0.7)$ & $2.8(1.1)$ & $3.4(0.8)$ & $3.2(1.2)$ \\
\hline $\begin{array}{l}\text { Splenic Conditions } \\
\text { (Spherocytosis) }\end{array}$ & $2.6(0.5)$ & $2.5(0.8)$ & $3.4(0.9)$ & $3.2(1.3)$ \\
\hline $\begin{array}{l}\text { Necrotizing Soft Tissue } \\
\text { Infections }\end{array}$ & $1.6(0.8)$ & $1.4(0.7)$ & $3.4(1.3)$ & $3.4(1.0)$ \\
\hline Intersex Abnormalities & $1.6(0.8)$ & $1.8(1.0)$ & $3.0(1.1)$ & $3.1(1.4)$ \\
\hline MANOVA & \multicolumn{2}{|l|}{$p=0.71 ; \mathrm{NS}$} & \multicolumn{2}{|l|}{$p=0.39 ; \mathrm{NS}$} \\
\hline
\end{tabular}

$\mathrm{NS}=$ Not significant 
Table 2: Pediatric Clinical and Surgical Skills Frequency and Importance Ratings by Residents and Staff $(n=35)$

\begin{tabular}{|c|c|c|c|c|}
\hline & Frequency Data & & Importance Da & \\
\hline $\begin{array}{l}\text { Pediatric Clinical and Surgical } \\
\text { Skills }\end{array}$ & $\begin{array}{l}\text { Staff Mean (SD) } \\
\mathrm{n}=\mathbf{2 3}\end{array}$ & $\begin{array}{l}\text { Resident Mean (SD) } \\
\mathrm{n}=12\end{array}$ & $\begin{array}{l}\text { Staff Mean (SD) } \\
n=23\end{array}$ & $\begin{array}{l}\text { Resident Mean (SD) } \\
\mathrm{n}=12\end{array}$ \\
\hline Vascular Access & $4.6(0.5)$ & $4.3(1.0)$ & $4.6(0.5)$ & $4.8(0.6)$ \\
\hline Advanced Laparoscopy & $4.3(0.9)$ & $4.8(0.5)$ & $4.2(0.9)$ & $4.9(0.3)$ \\
\hline Advanced Trauma Life Support & $3.8(0.9)$ & $4.3(0.6)$ & $4.7(0.5)$ & $4.7(0.5)$ \\
\hline Chest Tube Insertion & $3.8(0.7)$ & $3.5(0.7)$ & $4.5(0.7)$ & $4.4(0.7)$ \\
\hline Advanced Thoracoscopy & $3.4(0.9)$ & $3.8(0.5)$ & $4.0(1.0)$ & $4.8(0.5)$ \\
\hline $\begin{array}{l}\text { Clinical and Radiologic } \\
\text { Clearance of C-spine }\end{array}$ & $3.8(1.1)$ & $4.1(0.8)$ & $3.9(1.0)$ & $3.8(1.1)$ \\
\hline $\begin{array}{l}\text { Pediatric Advanced Life Support } \\
\text { (Skills) }\end{array}$ & $3.1(1.1)$ & $3.3(0.8)$ & $4.0(1.0)$ & $4.3(0.9)$ \\
\hline Upper Endoscopy & $3.3(1.2)$ & $3.2(1.0)$ & $3.6(1.1)$ & $3.7(1.1)$ \\
\hline Ultrasound Guided Procedures & $3.1(1.3)$ & $2.9(1.2)$ & $3.4(1.2)$ & $4.2(0.6)$ \\
\hline Trauma Laparotomy & $2.2(0.9)$ & $2.4(0.8)$ & $4.2(0.7)$ & $4.5(0.7)$ \\
\hline Bronchoscopy & $2.8(0.8)$ & $2.6(1.2)$ & $3.5(1.0)$ & $3.7(0.9)$ \\
\hline Dialysis Catheter Insertion & $2.9(0.9)$ & $2.8(1.1)$ & $3.4(1.0)$ & $3.3(1.3)$ \\
\hline Sigmoidoscopy & $2.9(1.1)$ & $2.6(1.1)$ & $3.4(1.0)$ & $3.3(1.3)$ \\
\hline FAST & $2.7(1.4)$ & $3.0(1.4)$ & $3.4(1.1)$ & $3.0(1.5)$ \\
\hline Endotracheal Intubation & $2.9(1.6)$ & $2.4(1.2)$ & $3.4(1.2)$ & $3.0(1.2)$ \\
\hline ECLS Cannulation & $2.2(1.3)$ & $2.5(1.2)$ & $3.0(1.2)$ & $4.2(1.1)$ \\
\hline Tracheostomy & $1.9(1.2)$ & $2.0(1.1)$ & $3.0(1.1)$ & $3.6(1.2)$ \\
\hline Intraosseous Needle Insertion & $1.8(0.9)$ & $1.8(1.1)$ & $3.5(1.0)$ & $3.2(1.2)$ \\
\hline Fasciotomy & $1.5(0.7)$ & $1.7(1.0)$ & $3.0(1.0)$ & $3.4(1.0)$ \\
\hline
\end{tabular}




\begin{tabular}{|l|l|l|l|l|}
\hline $\begin{array}{l}\text { Emergency Department } \\
\text { Thoracotomy }\end{array}$ & $1.1(0.3)$ & $1.3(0.5)$ & $2.7(1.4)$ & $3.3(1.2)$ \\
\hline MANOVA & $p=0.98 ; \mathrm{NS}$ & $p=0.50 ; \mathrm{NS}$ & \\
\hline
\end{tabular}

$\mathrm{NS}=$ Not significant 
Table 3: Pediatric Physiology Topic Frequency and Importance Ratings by Residents and Staff $(n=35)$

\begin{tabular}{|c|c|c|c|c|}
\hline \multirow[b]{2}{*}{ Pediatric Physiology Topics } & \multicolumn{2}{|c|}{ Frequency Data } & \multicolumn{2}{|l|}{ Importance Data } \\
\hline & $\begin{array}{l}\text { Staff Mean } \\
\text { (SD) } \quad n=23\end{array}$ & $\begin{array}{l}\text { Resident Mean (SD) } \\
\mathrm{n}=12\end{array}$ & $\begin{array}{l}\text { Staff Mean (SD) } \\
n=23\end{array}$ & $\begin{array}{l}\text { Resident Mean (SD) } \\
\mathrm{n}=12\end{array}$ \\
\hline Fluid and Electrolyte Management & $4.8(0.4)$ & $5.0(0.00)$ & $4.8(0.5)$ & $4.8(0.4)$ \\
\hline Nutrition & $4.7(0.6)$ & $4.6(1.2)$ & $4.5(0.6)$ & $4.8(0.5)$ \\
\hline Pediatric Pain & $5.0(0.0)$ & $4.6(1.2)$ & $4.5(0.6)$ & $3.9(1.2)$ \\
\hline Gastrointestinal Physiology & $4.3(0.7)$ & $4.6(0.5)$ & $4.0(0.9)$ & $4.2(0.8)$ \\
\hline Thermoregulation & $4.5(0.9)$ & $4.1(0.7)$ & $4.2(0.9)$ & $3.6(0.9)$ \\
\hline Shock & $3.6(0.8)$ & $3.9(0.5)$ & $4.7(0.4)$ & $4.7(0.5)$ \\
\hline $\begin{array}{l}\text { Transfusion Therapy and } \\
\text { Coagulation }\end{array}$ & $4.0(0.9)$ & $4.1(0.7)$ & $4.1(0.8)$ & $3.9(0.9)$ \\
\hline Pulmonary Physiology & $3.7(0.8)$ & $4.2(0.7)$ & $4.0(1.0)$ & $4.2(0.7)$ \\
\hline Cardiac Physiology & $3.5(.9)$ & $3.7(1.1)$ & $3.8(0.9)$ & $3.8(1.0)$ \\
\hline $\begin{array}{l}\text { Pediatric Advanced Life Support } \\
\text { (Physiology) }\end{array}$ & $2.7(1.3)$ & $3.1(0.9)$ & $4.1(1.0)$ & $4.0(1.0)$ \\
\hline Extra-Corporeal Life Support & $2.5(1.1)$ & $2.9(1.0)$ & $3.4(1.0)$ & $4.4(0.9)$ \\
\hline MANOVA & $p=0.54 ; \mathrm{NS}$ & & $p=0.70 ; \mathrm{NS}$ & \\
\hline
\end{tabular}

$\mathrm{NS}=$ Not significant 
Table 4: Rank Order Method Comparison Using Combined Data for Residents and Staff (N=35)

\begin{tabular}{|c|c|c|c|c|c|}
\hline \multirow[b]{2}{*}{$\begin{array}{l}\text { Rank (As per } \\
\text { Multiplicative } \\
\text { Model) }\end{array}$} & \multirow[b]{2}{*}{ Topic } & \multicolumn{4}{|c|}{ Rank Order Method } \\
\hline & & Frequency & Importance & $\begin{array}{l}\text { Multiplicative } \\
\text { Model }\end{array}$ & $\begin{array}{l}\text { Three-Step } \\
\text { Model }\end{array}$ \\
\hline 1 & $\begin{array}{l}\text { Fluid and Electrolyte } \\
\text { Management }\end{array}$ & 4.9 & 4.8 & 23.5 & 312 \\
\hline 2 & Appendicitis & 4.9 & 4.8 & 23.2 & 315 \\
\hline 3 & Pediatric Hernias & 4.9 & 4.8 & 23.2 & 309 \\
\hline 4 & Nutrition & 4.6 & 4.6 & 21.2 & 303 \\
\hline 5 & Pediatric Pain & 4.9 & 4.3 & 21.1 & 292 \\
\hline 6 & Vascular Access & 4.5 & 4.6 & 20.8 & 304 \\
\hline 7 & Advanced Laparoscopy & 4.4 & 4.5 & 19.7 & 291 \\
\hline 8 & Pediatric Burns and Trauma & 4.3 & 4.6 & 19.5 & 283 \\
\hline 9 & Pyloric Stenosis & 4 & 4.8 & 19.1 & 306 \\
\hline 10 & Necrotizing Enterocolitis & 3.8 & 4.9 & 18.7 & 285 \\
\hline 11 & Advanced Trauma Life Support & 4 & 4.7 & 18.5 & 288 \\
\hline 12 & Gastrointestinal Physiology & 4.4 & 4.1 & 18 & 276 \\
\hline 13 & Neonatal Bowel Obstruction & 3.6 & 4.8 & 17.5 & 267 \\
\hline 14 & Thermoregulation & 4.4 & 4 & 17.5 & 268 \\
\hline 15 & Shock & 3.7 & 4.7 & 17.4 & 273 \\
\hline 16 & Chest Tube Insertion & 3.7 & 4.5 & 16.5 & 266 \\
\hline 17 & Abdominal Wall Defects & 3.5 & 4.7 & 16.4 & 256 \\
\hline 18 & $\begin{array}{l}\text { Transfusion Therapy and } \\
\text { Coagulation }\end{array}$ & 4.1 & 4 & 16.3 & 267 \\
\hline 19 & Pulmonary Physiology & 3.9 & 4.1 & 15.9 & 254 \\
\hline
\end{tabular}




\begin{tabular}{|c|c|c|c|c|c|}
\hline 20 & Short Bowel Syndrome & 3.6 & 4.3 & 15.7 & 249 \\
\hline 21 & Intussusception & 3.5 & 4.4 & 15.4 & 261 \\
\hline 22 & Solid Malignancies & 3.5 & 4.4 & 15.3 & 246 \\
\hline 23 & $\begin{array}{l}\text { Esophageal Atresia/Trachea- } \\
\text { Esophageal Fistulas }\end{array}$ & 3.2 & 4.8 & 15.2 & 225 \\
\hline 24 & Advanced Thoracoscopy & 3.5 & 4.2 & 15 & 243 \\
\hline 25 & $\begin{array}{l}\text { Clinical and Radiologic } \\
\text { Clearance of C-spine }\end{array}$ & 3.9 & 3.9 & 15 & 230 \\
\hline 26 & $\begin{array}{l}\text { Anorectal Conditions and } \\
\text { Malformations }\end{array}$ & 3.2 & 4.7 & 14.9 & 222 \\
\hline 27 & $\begin{array}{l}\text { Congenital Diaphragmatic } \\
\text { Hernia }\end{array}$ & 3.1 & 4.8 & 14.9 & 216 \\
\hline 28 & Hirschsprungs Disease & 3.2 & 4.7 & 14.8 & 223 \\
\hline 29 & Cardiac Physiology & 3.6 & 3.8 & 13.7 & 239 \\
\hline 30 & $\begin{array}{l}\text { Congenital and Acquired Lung } \\
\text { Lesions }\end{array}$ & 3.1 & 4.3 & 13.6 & 216 \\
\hline 31 & Acute Scrotum & 3 & 4.4 & 13.4 & 217 \\
\hline 32 & Inflammatory Bowel Disease & 3.3 & 3.9 & 13.1 & 224 \\
\hline 33 & $\begin{array}{l}\text { Gastrointestinal and Airway } \\
\text { Foreign Bodies }\end{array}$ & 3.1 & 4.2 & 13 & 208 \\
\hline 34 & $\begin{array}{l}\text { Pediatric Advanced Life } \\
\text { Support (Skills) }\end{array}$ & 3.2 & 4.1 & 13 & 209 \\
\hline 35 & $\begin{array}{l}\text { Congenital/Acquired Neck } \\
\text { Lesions }\end{array}$ & 3.5 & 3.7 & 12.8 & 210 \\
\hline 36 & Chest Wall Defects & 3.5 & 3.7 & 12.8 & 192 \\
\hline 37 & Congenital Heart Anomalies & 3.7 & 3.3 & 12.2 & 191 \\
\hline
\end{tabular}




\begin{tabular}{|c|c|c|c|c|c|}
\hline 38 & Upper Endoscopy & 3.3 & 3.6 & 11.9 & 203 \\
\hline 39 & $\begin{array}{l}\text { Pediatric Gastrointestinal } \\
\text { Bleeding }\end{array}$ & 3 & 3.8 & 11.5 & 200 \\
\hline 40 & Pediatric Obesity & 3.5 & 3.3 & 11.4 & 174 \\
\hline 41 & $\begin{array}{l}\text { Pediatric Advanced Life } \\
\text { Support (Physiology) }\end{array}$ & 2.8 & 4.1 & 11.4 & 186 \\
\hline 42 & Genitourinary Conditions & 3.4 & 3.3 & 11.2 & 186 \\
\hline 43 & Ultrasound Guided Procedures & 3 & 3.7 & 11.1 & 189 \\
\hline 44 & Hematologic Malignancies & 3.6 & 3 & 10.8 & 190 \\
\hline 45 & $\begin{array}{l}\text { Congenital Hepatobiliary } \\
\text { Abnormalities }\end{array}$ & 2.5 & 4.2 & 10.5 & 164 \\
\hline 46 & $\begin{array}{l}\text { Extra-Corporeal Life Support } \\
\text { (Physiology) }\end{array}$ & 2.7 & 3.8 & 10.2 & 176 \\
\hline 47 & Trauma Laparotomy & 2.3 & 4.3 & 9.9 & 147 \\
\hline 48 & Bronchoscopy & 2.7 & 3.6 & 9.8 & 163 \\
\hline 49 & Dialysis Catheter Insertion & 2.9 & 3.4 & 9.7 & 166 \\
\hline 50 & Sigmoidoscopy & 2.8 & 3.4 & 9.5 & 157 \\
\hline 51 & FAST & 2.8 & 3.3 & 9.3 & 158 \\
\hline 52 & Gynecologic Conditions & 2.9 & 3.2 & 9.2 & 162 \\
\hline 53 & Endotracheal Intubation & 2.7 & 3.3 & 8.9 & 153 \\
\hline 54 & $\begin{array}{l}\text { Splenic Conditions } \\
\text { (Spherocytosis) }\end{array}$ & 2.6 & 3.2 & 8.1 & 138 \\
\hline 55 & $\begin{array}{l}\text { Extra-Corporeal Life Support } \\
\text { Cannulation }\end{array}$ & 2.3 & 3.4 & 7.9 & 147 \\
\hline 56 & Tracheostomy & 1.9 & 3.2 & 6.1 & 113 \\
\hline 57 & Intraosseous Needle Insertion & 1.8 & 3.4 & 6 & 101 \\
\hline
\end{tabular}




\begin{tabular}{|l|l|r|r|r|r|}
$\mathbf{5 8}$ & Necrotizing Soft Tissue & 1.5 & 3.4 & 5.3 & 92 \\
\hline $\mathbf{5 9}$ & Infections & & & \\
\hline $\mathbf{6 0}$ & Intersex Abnormalities & 1.7 & 3.1 & 5.1 & 95 \\
\hline $\mathbf{6 1}$ & Fasciotomy & 1.6 & 3.1 & 4.9 & 90 \\
& Emergency Department & 1.2 & 2.9 & 3.4 & 69 \\
\hline
\end{tabular}

Note: The topics are listed in order as by the ranking from the multiplicative model from highest to lowest. 
CHAPTER 3: CONCLUSION 


\subsection{Summary of Main Findings}

Residents entering into pediatric surgery training programs face unique challenges as they adapt to a new patient population whilst developing their subspecialty skill set. Through a needs assessment survey, key stakeholders (i.e., residents and staff) in pediatric surgery were found to share similar views on critical content areas that should be covered in a pediatric surgery boot camp, designed to ease the transition into subspecialty training. There was agreement that the primary focus of the boot camp should be on pediatric surgical diagnoses, such as appendicitis and pediatric hernias, as well as pediatric physiology, including topics like fluid and electrolyte management and nutrition. For the most part surgical and clinical skills training was felt to be less critical for inclusion in a pediatric surgery boot camp, with only a few skills ranking highly overall, such vascular access and advanced laparoscopy.

Although the content section of the survey primarily focused on medical expert competencies under the assumption that pediatric surgery residents would have attained competence in the non-medical expert roles during adult surgical training, the open-ended section allowed survey participants to identify additional competencies that were felt relevant for inclusion in a pediatric surgery boot camp. Despite the overlap between adult and pediatric surgical training in these non-medical expert competencies, certain unique circumstances were identified by survey particpants, including communication with difficult pediatric patients and anxious parents, or patient advocacy in the setting of child abuse.

Lastly, the preferred duration of a pediatric surgery boot camp was determined to be short and focused, in the range of 3-4 days, encompassing tailored educational methods like elearning, small group teaching, didactic lectures and problem based learning. 


\subsection{Study Limitations}

Utilizing a needs assessment survey to guide the development of a pediatric surgery boot camp does provide some limitations. First and foremost, the overall number of completed surveys was low. To maximize the number of surveys returned, incentives were offered in the form of online gift cards and the survey was designed to take less than 15 minutes to complete. Furthermore, reminder emails were provided to participants in an attempt to maximize response rate.

Despite the low number of completed surveys, the number equates to a completion rate of almost $20 \%$ of the entire population of staff and learners in pediatric surgery at academic centres across Canada and the United States. Participants most likely to complete the survey were those closely involved in post graduate medical education and resident training: program directors and pediatric surgery residents. This type of selection bias would only serve to enhance our results given that this population is more in tune with the needs of current residents, and therefore more likely to rate items appropriately on the level of importance as it pertains to new residents and the frequency in which they are encountered by new residents.

The survey nature of the study also limited the ability to ask further questions to respondents after survey completion, or to clarify responses in the open ended question sections. Alternative methods of gathering data, such as formal interviews or focus groups, would allow for answer clarification and more in-depth, open responses. However, given the wide distribution of targeted learners and pediatric surgery staff across Canada and the United States, these methods were not feasible due to cost of travel and study time constraints. Thus, electronic administration ensured the survey could be distributed to the largest number of participants with the lowest overall cost. For future endeavours, to optimize survey design and 
outcomes, one could consider taking advantage of national meetings, such as the annual meeting for CAPS, to perform focus group interviews in advance to survey creation and distribution. This would enable survey development to be tailored to emergent issues from the focus group discussions. Furthermore, by engaging participants in person at a national meeting, buy-in for survey completion may be improved.

Lastly, despite the care and planning that went into survey development and design, online survey questions may appear ambiguous to some respondents, with no means available for clarification. To help ensure clear and unambiguous questions, as well as to ensure that it gathered all of the pertinent data required for the development of a pediatric surgery boot camp, the survey was developed in conjunction with a pediatric surgeon, resident and curriculum design expert. Following its development, the survey was piloted to a small test group of pediatric surgery residents and staff.

\subsection{Directions of Future Research}

As was demonstrated in our meta-analysis, [31] boot camps have been shown to be an effective tool for improving learner skills, knowledge and confidence during critical transition periods in medical education. Building upon this idea, subsequent phases of our research program will include development and pilot testing of a pediatric surgery boot camp using the information gathered from our study. The rank order lists will help guide curricular content, while the format preferences will help structure the boot camp to meet the needs of the trainee. This boot camp will be formatted using the Kern model for curricular design, with a large focus on assessment and evaluation, both of the learner and the curriculum.[44]

Ongoing evaluation will help in ensuring the curriculum achieves its goals and can adapt to the needs of the learners and staff over time. Part of this ongoing evaluation would include 
assessment of learner knowledge and skills, as well as learner stress and well being during the transition to pediatric surgery, both before and after taking part in a surgical boot camp. In the long term, after the implementation of a pediatric surgery boot camp, patient outcomes at academic pediatric surgical centres could be reassessed through administrative database analysis to identify if any trends towards improved patient outcomes develop.

\subsection{Patient and Learner Implications}

In a response to changing patient needs, greater patient expectations for high-quality care, and increasing work hour restrictions for trainees, the Competence by Design Initiative from the RCPSC made recommendations to help improve patient safety, reduce learner stress and improve productivity during critical transition periods in medical education. These recommendations included a greater focus during the transition to discipline specific residency programs. This can be achieved through the implementation of courses designed to orient and assess new trainees, and ensuring key competencies are achieved prior to involvement in patient care. $[20,34]$

In keeping with these recommendations, the aim of creating and implementing this boot camp would be to improve new residents' confidence, to increase their knowledge of key pediatric surgical topics, and to sharpen their pediatric surgical and clinical skills during the transition from general surgery to pediatric surgery. We anticipate this can be achieved through early exposure to the topics and skills deemed critical for transition into pediatric surgery, and by providing opportunities to apply knowledge and practice these skills in a setting where immediate formative feedback is available. By ensuring competence in these areas through observation and evaluation, we anticipate potential benefits in patient care if improved knowledge, and skill lead to lower patient morbidity and mortality.[45-49] 
Furthermore, by exposing learners to the common and critical pediatric surgical diagnoses, physiological topics, and skills at the start of their training, we hope to bring learners to common ground and increase their confidence with regards to these key competencies. If the improved confidence in dealing with these topics equates to lower levels of perceived stress as demonstrated in previous studies, a pediatric surgery boot camp may also contribute to lower levels of psychiatric morbidity and burnout amongst new trainees.[9,50] In addition to the above benefits to learners and patients, by providing a venue for early interaction between residents and staff from multiple pediatric surgery programs nationwide, a boot camp may also provide opportunities for improved collaborative efforts and career networking. Such opportunities are invaluable in a specialty like pediatric surgery where centres, often isolated by large geographical distances, may see as few as one or two trainees during any given training period.

To summarize, the benefits of a pediatric surgical boot camp start with improved confidence, knowledge and skills amongst new trainees. However, these benefits do not end when the boot camp finishes as future benefits, while vast and difficult to capture, include improved patient outcomes, decreased learner stress and burnout, and the creation of networking opportunities. 


\section{REFERENCES}

1. Nishisaki A, Hales R, Biagas K, et al. A multi-institutional high-fidelity simulation "boot camp" orientation and training program for first year pediatric critical care fellows. Ped Crit Care Med. 2009;10(2):157-162.

2. Van Hell EA, Kuks JB, Borleffs JC, Cohen-Schotanus J. Alternating skills training and clerkships to ease the transition from preclinical to clinical training. Med Teach. 2011;33(12):e689-96.

3. Radcliffe C, Lester H. Perceived stress during undergraduate medical training: A qualitative study. Med Educ. 2003;37(1):32-38.

4. Parent RJ, Plerhoples TA, Long EE, et al. Early, intermediate, and late effects of a surgical skills "boot camp" on an objective structured assessment of technical skills: A randomized controlled study. J Am Coll Surg. 2010;210(6):984-989.

5. Nakayama DK, Steiber A. Surgery interns' experience with surgical procedures as medical students. Am J Surg. 1990;159(3):341-344.

6. Brunt LM, Halpin VJ, Klingensmith ME, et al. Accelerated skills preparation and assessment for senior medical students entering surgical internship. J Am Coll Surg. 2008;206(5):897-904 
7. Esterl RM,Jr, Henzi DL, Cohn SM. Senior medical student "boot camp": Can result in increased self-confidence before starting surgery internships. Curr Surg. 2006;63(4):264-268.

8. Jellinek MS, Todres ID, Catlin EA, Cassem EH, Salzman A. Pediatric intensive care training: Confronting the dark side. Crit Care Med. 1993;21(5):775-779.

9. Willcock SM, Daly MG, Tennant CC, Allard BJ. Burnout and psychiatric morbidity in new medical graduates. Med J Aust. JID - 0400714. 1201(0025-729; 0025-729).

10. van Walraven C, Jennings A, Wong J, Forster AJ. Influence of house-staff experience on teaching-hospital mortality: The "July phenomenon" revisited. J Hosp Med. 2011;6(7):389-394.

11. Ehlert BA, Nelson JT, Goettler CE, et al. Examining the myth of the "July Phenomenon" in surgical patients. Surgery. 2011;150:332-338.

12. Weaver KJ, Neal D, Hoh DJ, et al. The "July Phenomenon" for neurosurgical mortality and complications in teaching hospitals: An analysis of more than 850000 neurosurgical patients in the Nationwide Inpatient Sample database, 1998 to 2008. Neurosurgery. 2012;71:562-571.

13. Schroeppel TJ, Fischer PE, Magnotti LJ, et al. The "July Phenomenon": Is trauma the exception? J Am Coll Surg. 2009;209:378-384. 
14. Inaba K, Recinos G, Teixeira PG, et al. Complications and death at the start of the new academic year: Is there a july phenomenon? $J$ Trauma. 2010;68(1):19-22

15. Jen MH, Bottle A, Majeed A, Bell D, Aylin P. Early in-hospital mortality following trainee doctors' first day at work. PLoS ONE 4(9): e7103. doi:10.1371/journal.pone.0007103

16. Dasenbrock HH, Clarke MJ, Thompson RE, et al. The impact of July hospital admission on outcome after surgery for spinal metastases at academic medical centers in the United States, 2005 to 2008. Cancer. 2012;118:1429-1438.

17. Phillips DP, Barker GEC. A July spike in fatal medication errors: A possible effect of new medical residents. J Gen Intern Med. 2010;25(8):774-779.

18. Young JQ, Ranji SR, Wachter RM, et al. "July Effect": Impact of the academic year-end changeover on patient outcomes. Ann Intern Med. 2011;155:309-315.

19. Sonnadara RR, Garbedian S, Safir O, et al. Orthopaedic boot camp II: Examining the retention rates of an intensive surgical skills course. Surgery. 2012;151(6):803-807.

20. Lockyer J, Silver I, Oswald A, et al. The continuum of medical education. In: Competence by design: Reshaping Canadian Medical Education. Vol 2014. http://www.royalcollege.ca/portal/page/portal/rc/common/documents/educational_initiatives/rc_ competency-by-design_ebook_e.pdf ed. ; 2014:130-141. 
21. Iobst WF, Sherbino J, Cate OT, et al. Competency-based medical education in postgraduate medical education. Med Teach. 2010;32(8):651-656.

22. Polavarapu HV, Kulaylat AN, Sun S, Hamed OH. 100 years of surgical education: the past, present and future. Bull Am Coll Surg. 2013;98(7):22-27.

23. Grillo HC. To impart this art: The development of graduate surgical education in the United States. Surgery. 1999;125(1):1-14.

24. Ericsson KA. Deliberate practice and acquisition of expert performance: A general overview. Acad Emerg Med. 2008;15(11):988-994.

25. McGaghie WC, Issenberg SB, Cohen ER et al. Does simulation-based education with deliberate practice yield better results than traditional clinical education? A meta-analytic comparative review of the evidence. Acad Med. 2011;86:706-711.

26. McGaghie WC, Issenberg SB, Barsuk JH, Wayne DB. A critical review of simulation-based mastery learning with translational outcomes. Med Educ. 2014;48(4):375-385.

27. McGaghie WC, Draycott TJ, Dunn WF et al. Evaluating the impact of simulation on translational patient outcomes. Simul Healthc. 2011;6 (3):42-47. 
28. McGaghie WC, Issenberg SB, Cohen ER et al. Translational educational research: a necessity for effective health care improvement. Chest. 2012;142: 1097-1103.

29. Santen SA, Deiorio NM, Gruppen LD. Medical education research in the context of translational science. Acad Emerg Med. 2012;19:1323-1327.

30. Cohen ER, Feinglass J, Barsuk JH et al. Cost savings from reduced catheter-related bloodstream infection after simulation-based education for residents in a medical intensive care unit. Simul Healthc. 2010;5:98-102

31. Blackmore C, Austin J, Lopushinsky S, Donnon T. The effects of postgraduate medical education "Boot camps" on clinical skills, knowledge and confidence: A meta-analysis. J Grad Med Ed. 2014;6(4):643-652.

32. DerSimonian R, Laird N. Meta-analysis in clinical trials. Control Clin Trials. 1986;7(3):177188.

33. Matlow AG, Baker GR, Flintoft VF, et al. Adverse events among children in Canadian hospitals: The Canadian paediatric adverse events study. CMAJ : Canadian Medical Association journal = journal de l'Association medicale canadienne JID - 9711805. 1128.

34. Snell L, Frank JR, Stoneham G, et al. Competency-based medical education. In: Competence by design: Reshaping canadian medical education. 2014: 99-105.

http://www.royalcollege.ca/portal/page/portal/rc/common/documents/educational_initiatives/rc_ competency-by-design_ebook_e.pdf. 
35. Holmboe ES, Edgar L. Next accredidation system: Milestones.

https://www.acgme.org/acgmeweb/tabid/430/ProgramandInstitutionalAccreditation/NextAccredi tationSystem/Milestones.aspx. Updated 2014. Accessed 06/27, 2014.

36. Selden NR, Origitano TC, Burchiel KJ, et al. A national fundamentals curriculum for neurosurgery PGY1 residents: The 2010 society of neurological surgeons boot camp courses. Neurosurgery. 2012;70(4):971-981.

37. Sonnadara RR, Van Vliet A, Safir O, et al. Orthopedic boot camp: Examining the effectiveness of an intensive surgical skills course. Surgery. 2011;149(6):745-749.

38. Antonoff MB, Shelstad RC, Schmitz C, Chipman J, D'Cunha J. A novel critical skills curriculum for surgical interns incorporating simulation training improves readiness for acute inpatient care. J Surg Educ. 2009;66(5):248-254.

39. The Royal College of Physicians and Surgeons of Canada. Objectives in training in the subspecialty of pediatric surgery. http://rcpsc.medical.org/residency/certification/objectives/pedgen-surgery e.pdf. Updated 2012. Accessed 11/25, 2012.

40. The Accrediation Council for Graduate Medical Education. The Pediatric Surgery Milestone Project.

https://www.acgme.org/acgmeweb/Portals/0/PDFs/Milestones/PediatricSurgeryMilestones.pdf. Updated February 2014. Accessed 08/26, 2014. 
41. McLaughlin K, Lemaire J, Coderre S. Creating a reliable and valid blueprint for the internal medicine clerkship evaluation. Med Teach. 2005;27(6):544-547.

42. Smith SD, Beran TN. A comparison of empirical ranking methods of frequency and severity ratings of clinical presentations. Eval Health Prof. 2012;35(4):383-395.

43. Kaplowitz MD, Hadlock TD, Levine R. A comparison of web and mail survey response rates. Public Opin Q. 2004; 68(1): 94-101.

44. Kern DE, Thomas PA, Hughes MT. Curriculum development for medical education. Second ed. Baltimore: The Johns Hopkins University Press; 2009.

45. Cohen ER, Barsuk JH, Moazed F, et al. Making July safer: simulation-based master learning during inter boot camp. Acad Med. 2013;88:233-239.

46. Barsuk JH, McGaghie WC, Cohen ER, et al. Simulation-based mastery learning reduces complications during central venous catheter insertion in a medical intensive care unit. Crit Care Med. 2009;37:2697-2701.

47. Wayne DB, Didwania A, Feinglass J, et al. Simulation based education improves quality of care during cardiac arrest team responses at an academic teaching hospital: A case-control study. Chest. 2008;133:56-61. 
48. Barsuk JH, Cohen ER, Feinglass J, et al. Use of simulation-based education to reduce catheter-related bloodstream infections. Arch Intern Med. 2009;169:1420-1423.

49. Butter J, McGaghie WC, Cohen ER, Kaye M, Wayne DB. Simulation-based mastery learning improves cardiac auscultation skills in medical students. J Gen Intern Med. 2010;25:780-785.

50. Mueller G, Hunt B, Wall V, et al. Intensive skills week for military medical students increases technical proficiency, confidence, and skills to minimize negative stress. $J$ Spec Oper Med. 2012;12(4):45-53 
APPENDIX A: BOOT CAMP SYSTEMATIC REVIEW AND METANALYSIS WITH PERMISION FOR USE FROM JOURNAL 


\title{
Effects of Postgraduate Medical Education "Boot Camps" on Clinical Skills, Knowledge, and Confidence: A Meta-Analysis
}

CHRISTOPHER BLACKMORE, MD

JANICE AUSTIN, MD

SteVEn R. LOPUSHINSKY, MD, MSC

TYRONE DONNON, PHD

\begin{abstract}
Background Throughout their medical education, learners face multiple transition periods associated with increased demands, producing stress and concern about the adequacy of their skills for their new role.

Objective We evaluated the effectiveness of boot camps in improving clinical skills, knowledge, and confidence during transitions into postgraduate or disciplinespecific residency programs.

Methods Boot camps are in-training courses combining simulation-based practice with other educational methods to enhance learning and preparation for individuals entering new clinical roles. We performed a search of MEDLINE, CINAHL, PsycINFO, EMBASE, and ERIC using boot camp and comparable search terms. Inclusion criteria included studies that reported on medical education boot camps, involved learners entering new clinical roles in North American programs,

and reported empirical data on the effectiveness of boot camps to improve clinical skills, knowledge, and/or confidence. A random effects model meta-analysis was performed to combined mean effect size differences (Cohen's $d$ ) across studies based on pretest/posttest or comparison group analyses.

Results The search returned 1096 articles, 15 of which met all inclusion criteria. Combined effect size estimates showed learners who completed boot camp courses had significantly "large" improvements in clinical skills $(d=$ 1.78; $95 \% \mathrm{Cl} 1.33-2.22 ; P<.001)$, knowledge $(d=2.08$; $95 \% \mathrm{Cl} 1.20-2.96 ; P<.001)$, and confidence $(d=1.89$; $95 \% \mathrm{Cl} 1.63-2.15 ; P<.001)$.

Conclusions Boot camps were shown as an effective educational strategy to improve learners' clinical skills, knowledge, and confidence. Focus on pretest/posttest research designs limits the strength of these findings.
\end{abstract}

\section{Introduction}

Throughout undergraduate and postgraduate medical education, learners are faced with a series of transitions that can be stressful, which can raise questions about the adequacy of their skills for their new role. For example, the transition from medical school to postgraduate training or into a discipline-specific subspecialty residency program (ie,

All authors are in the Faculty of Medicine at the University of Calgary, Calgary, Alberta, Canada. Christopher Blackmore, MD, is General Surgery Resident, Department of Surgery; Janice Austin, MD, is General Surgery Resident Department of Surgery; Steven R. Lopushinsky, MD, MSc, is Clinical Assistant Professor, Department of Pediatric Surgery; and Tyrone Donnon, PhD, is Associate Professor, Medical Education and Research Unit, Department of Community Health Sciences

Funding: The authors report no external funding source for this study. Conflict of interest: The authors declare they have no competing interests.

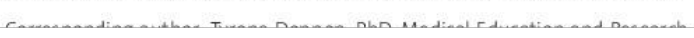

Received October 18, 2013; revisions received April 28, 2014, and July 21, 2014: accepted July 21, 2014.

DOI: http://dx.doi.org/10.4300/JGME-D-13-00373.1 junior to senior) can be stressful because of the variability of teaching and learning opportunities provided for clinical or procedural skills training as well as the increased expectations in learners' clinical responsibilities. ${ }^{1-4}$ Similarly, the transition from residency to fellowship has been cited as a time of great stress given the increased complexity of patients' needs, their personal sense of responsibility, and their expectations of clinical performance without failure.5,6 Furthermore, these periods have also been associated with increased rates of psychiatric morbidity and burnout among trainees ${ }^{7}$ and a growing concern for the care and safety of the patient, giving rise to terms like July phenomenon or July effect. 8,9

Concerns are amplified under current duty hour restrictions in effect for trainees. Less time spent learning clinical and procedural skills can lead to a decrease in learner competence and patient safety. For instance, Poulouse et $\mathrm{al}^{10}$ found a greater rate of needle injuries after work hour restrictions, suggesting reduced clinical experiences lead to less proficiency on some procedural tasks. Given the medicolegal concerns associated with medical errors because of work hour restrictions and transition 
periods, there has been a strong push toward introducing new educational strategies within clinical training programs to potentially mitigate those effects. ${ }^{11}$

Before the development of competency-based education frameworks, teaching and learning for most specialties took the form of a master-apprentice relationship in which trainees observed their clinical mentors in practice. As learners became more familiar with the knowledge and skills of their specialty, they began to perform more intricate tasks with growing independence. ${ }^{11,12}$ According to the Ericsson ${ }^{13}$ theory of how expertise is achieved, active engagement that involves deliberate practice and immediate feedback is essential in the acquisition of knowledge and the ability to perform. A recent meta-analysis comparing traditional to simulation-based medical education with deliberate practice supported the superiority of this approach to enhancing learners' clinical knowledge and skills. ${ }^{14}$ Using this theoretic principle as a guide to enhance clinical expertise, many medical education programs across North America have adopted the concept of deliberate practice and adapted it in "boot camp" courses specifically designed to ease learners into new clinical roles during transition periods.

Despite the increasing popularity of boot camps in medical education, there is no prior review on their effectiveness published in the literature. The objective of this study was to perform a descriptive analysis of boot camps in medical education and a meta-analysis to assess the effectiveness of boot camp courses as an educational strategy to prepare learners during transition periods in clinical training programs. The main focus was to assess the effectiveness of boot camps across 3 important areas: clinical skills performance, knowledge acquisition, and confidence in clinical abilities.

\section{Methods}

\section{Study Selection}

In consultation with a medical librarian, we conducted a systematic review of the research on the effectiveness of boot camps published between January 1995 and April 2013 using MEDLINE, CINAHL, PsycINFO, EMBASE, and ERIC. For the purpose of study selection, boot camps were defined as courses designed to enhance learning and preparation for those entering new clinical roles with simulation-based practice and other related educational strategies. Initial identification of search terms was based on literature and articles on medical education boot camps. Terms were identified through literature review to encompass all synonyms used to describe boot camp courses, including crash course, capstone course, transition course, or rookie camp. A review of references for all included articles was performed to identify any additional studies for inclusion in our review and analysis. The search was further limited to the heading medical education. Two authors (C.B. and J.A.) independently retrieved and reviewed articles for study selection and also reviewed the references of selected articles to identify additional reports. All conflicts in article selection were resolved by consensus.

\section{Eligibility Criteria}

Studies were included if they (1) reported on medical education boot camps for trainees transitioning from medical school or already in a residency program in North America, (2) involved trainees who were expecting to enter into new clinical roles, (3) assessed the effectiveness of the boot camp session(s) pretest/posttest (ie, immediately after the boot camp intervention) or with a comparison group, and (4) provided empirical data on clinical skills, knowledge acquisition, and/or confidence measures. The authors restricted the search to North American studies to ensure that different formats of boot camp courses reflected similar clinical transition periods for medical students and residents. For this study, residents who completed preliminary training were considered to have been provided with a boot camp to assist with transition into discipline-specific training.

\section{Data Selection and Abstraction}

To address concerns about bias, we conducted a comprehensive search using strict selection criteria based on rigorous interrater reliability. Two authors (C.B. and J.A.) were involved in independently reviewing abstracts and coding the data from the full-text articles identified. The following data were collected: year of study, specialties involved, sample size, level of training of the participants, duration of the boot camp, type of study research design, boot camp design and definition, outcome variables assessed, and the measured empirical values reported, including means and standard deviations.

Statistical analysis was performed using Stata version 12.1 (StataCorp LP). All data from the outcome measures were continuous, and the meta-analysis was performed using sample means and standard deviations to calculate the Cohen's $d$ for effect size differences. ${ }^{15}$ For studies where standard deviations were not provided, we used the $P$ values from the $t$ test statistic to estimate the comparable effect size difference. ${ }^{16}$ We chose the random effects model for our meta-analysis based on the variability among studies in length of boot camp session(s), content delivered, evaluations used, and levels of transition for the different groups' participants. ${ }^{17}$ The interpretation of the magnitude of the combined effect sizes were based on Cohen's ${ }^{15}$ suggestions: $d=0.20$ to 0.49 are small, $d=0.50$ to 0.79 
are medium, and $d \geq 0.80$ are considered to be large effect size differences. The heterogeneity of the combined study outcomes were tested using the $I^{2}$ statistic. To estimate the between-studies variance, an $I^{2}$ value of $50 \%$ implies that half of the observed variability can be attributed to between-studies variance (heterogeneity in the boot camp interventions) and the other half within-study variance (ie, sampling error)..$^{18}$ To minimize study heterogeneity, studies comparing pretest/posttest scores for boot camp participants were analyzed separately from studies using comparisons or control groups.

\section{Results}

The literature search identified 1096 articles. After screening, 36 full-text articles were assessed for eligibility. Of those 36 articles, 21 were excluded because they failed to provide sufficient empirical data, did not pertain to boot camps, reported duplicate data, or were not based in medical education or from North America. Fifteen studies met all inclusion criteria. ${ }^{1,3,19-31}$ Those 15 articles underwent coding extraction independently by 2 authors (C.B. and J.A.) to ensure data collection consistency and accuracy of effect size calculation analyses. Any discrepancies were reviewed by the other authors until agreement was achieved.

\section{Descriptive Review}

From the 15 studies included in our meta-analysis, a range of definitions for boot camps were used (T A B LE 1). Despite differences in wording, most describe a boot camp as an early preparatory course or orientation sessions for learners undergoing a transition in medical education. The design and development of these boot camps varied from an informal curricular design by surgical staff ${ }^{19}$ to a formal needs assessment approach to understanding clinical skill development. ${ }^{26}$ Although a variety of educational methods and teaching strategies were used, every boot camp used some form of low- or high-technology simulation as a key component. In addition, 10 of 15 boot camps $(67 \%)$ described providing either immediate or formative feedback to trainees ( 3 boot camps did not specify whether feedback was given, and 2 boot camps engaged only in debriefing sessions following simulation and case scenarios).

Regarding the particular medical education transitions targeted by the boot camps, we identified that studies focused on either the transition from medical school to graduate/postgraduate education ( 6 of $15,40 \%$ ) or into a discipline-specific (eg, specialty/subspecialty) residency program ( 9 of $15,60 \%$; T A B LE 2). Only 1 specific boot camp for fellows was identified in our search, and it provided insufficient data for statistical analysis. ${ }^{5}$ The number of boot camp participants ranged from 6 to 47 , and participants came from a variety of specialties, including internal medicine, general surgery, obstetrics and gynecology, cardiac surgery, thoracic surgery, and orthopedic surgery. Fourteen of $15(93 \%)$ studies involved surgical specialties or subspecialties (TABLE 2). For boot camps involving medical students at the end of their clerkship period, students had applied or were matched to surgical specialties. The length of time of the boot camp varied from 4 hours (over 2.5 days) ${ }^{23}$ to 160 hours (over 30 days) 27 $^{27}$ and was completed in a single day ( 8 hours) for residents transitioning into otolaryngology ${ }^{26}$ or was spread across a 7 -week period ( 2 to 3 hours per week) for medical students beginning their surgery residency. ${ }^{3}$

The majority of studies used a pretest/posttest (13 of $15,87 \%$ ) assessment process to determine the effectiveness of the boot camp intervention immediately on completion (T A B LE 2). Four studies also included a comparison group..$^{3,19,27,30}$ One study assessed boot camp participants' performance using only a comparison group who had not completed the boot camp, ${ }^{1}$ and 1 study compared participants only to historic controls. ${ }^{31}$ Three sets of learner outcome measures were identified and, if reported, were combined across studies: (1) clinical skills performance $(6$ of $15,40 \%),(2)$ knowledge acquisition (4 of $15,26 \%)$, and (3) participants' confidence in their clinical abilities ( 8 of $15,53 \%)$.

\section{Clinical Skills Performance}

Six boot camp studies (40\%) provided pretest/posttest data on trainees' performance on different clinical skills, using either task-specific checklist scores or global rating scales, such as an Objective Structured Assessment of Technical Skills. ${ }^{32}$ Although some of the clinical performances assessed were related to the overall completion of the surgical or procedural skill itself (eg, central line insertion, chest tube insertion, lumbar puncture), many outcome measures were related to generic skills important for successful clinical practice (eg, restricted space tying, suturing). As shown in FIGURE 1, 16 clinical skill outcomes were combined across the 6 studies for a large effect size of $d=1.78$ (95\% CI 1.33-2.22, $P<.001)$. The heterogeneity was found to be moderately high with an $I^{2}$ statistic value of $81.2 \%(P<.01)$. A reported randomeffects model for combined effect sizes is recommended when the heterogeneity is above $50 \%$.

TABLE 3 shows a combined random-effects model for the 4 studies ( $27 \%, 9$ outcomes) where boot camp trainees' clinical skills performance scores were contrasted with a comparison group, showing a large effect size difference of $d=2.73(95 \%$ CI $1.36-4.07, P<.001)$. 


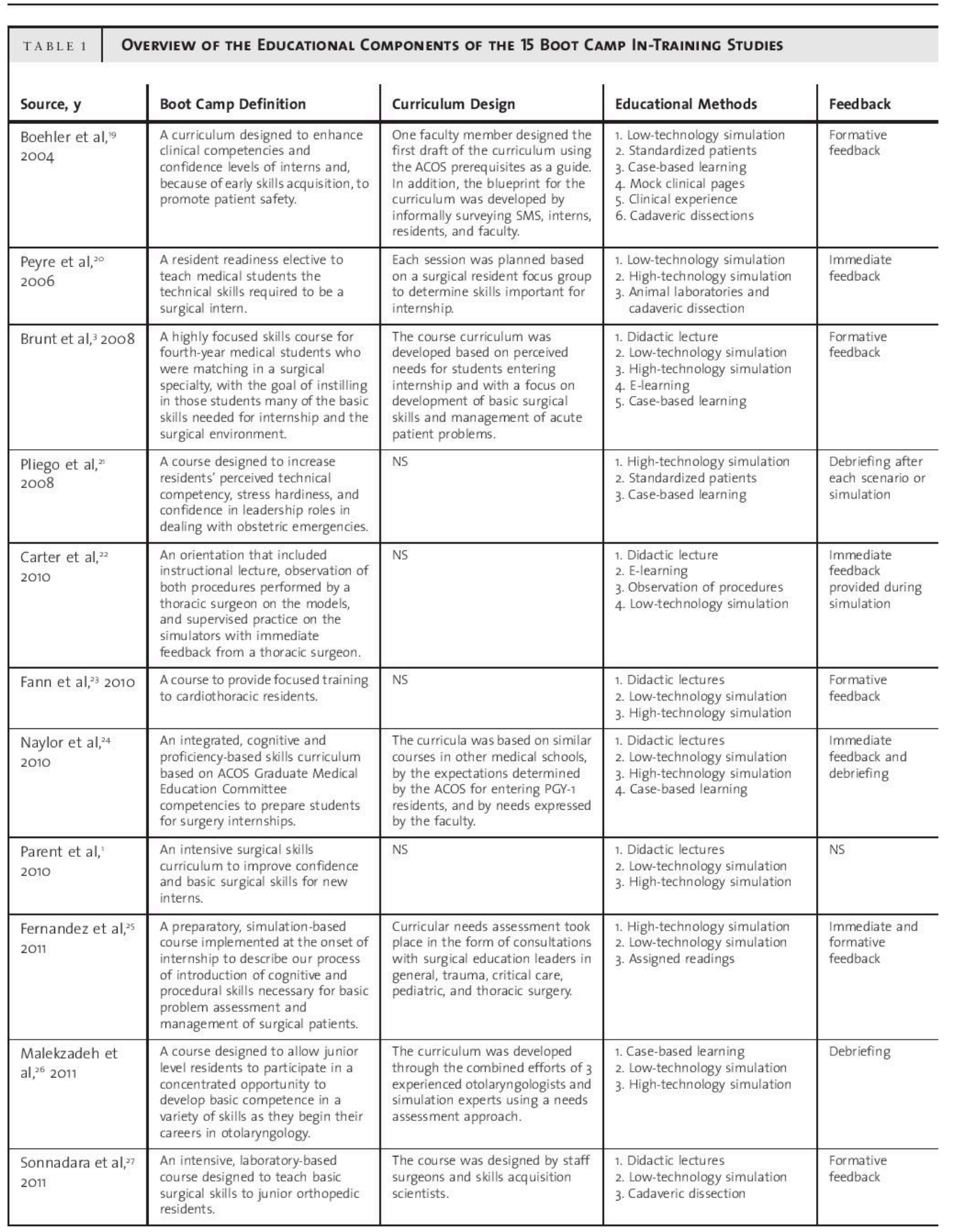

646 Journal of Graduate Medical Education, December 2014 


\begin{tabular}{|c|c|c|c|c|}
\hline TABLE 1 & RVIEW OF THE EdUCATIONAL CO & MPONENTS OF THE 15 BOOT C & MP IN-TRAINING STUdIES & JTINUED) \\
\hline Source, $y$ & Boot Camp Definition & Curriculum Design & Educational Methods & Feedback \\
\hline Todd et al, ${ }^{28} 2011$ & $\begin{array}{l}\text { A surgical intern survival skills } \\
\text { curriculum designed to alleviate } \\
\text { anxiety through a series of lectures } \\
\text { and interactive sessions. }\end{array}$ & NS & $\begin{array}{l}\text { 1. Small group discussion } \\
\text { 2. Didactic lectures } \\
\text { 3. Mock clinical pages }\end{array}$ & $\begin{array}{l}\text { Immediate } \\
\text { feedback }\end{array}$ \\
\hline $\begin{array}{l}\text { Antonoff et al, }{ }^{29} \\
2011\end{array}$ & $\begin{array}{l}\text { A comprehensive PGY-1 surgery } \\
\text { preparatory course offered to senior } \\
\text { medical students matched into } \\
\text { residencies in surgical disciplines. }\end{array}$ & $\begin{array}{l}\text { Learning objectives for the course } \\
\text { were generated by a committee } \\
\text { consisting of clinical surgical } \\
\text { faculty members and residents, } \\
\text { with input from a previously } \\
\text { established internal curriculum } \\
\text { for PGY-1 residents. }\end{array}$ & $\begin{array}{l}\text { 1. Interactive lecture } \\
\text { 2. Small group sessions } \\
\text { 3. High-technology simulation } \\
\text { 4. Low-technology simulation } \\
\text { 5. Problem-based learning } \\
\text { 6. Mock clinical pages }\end{array}$ & NS \\
\hline $\begin{array}{l}\text { Okusyana et al, },^{30} \\
2012\end{array}$ & $\begin{array}{l}\text { A course incorporating technical } \\
\text { and medical management skills } \\
\text { designed to ensure that interns } \\
\text { achieve a baseline competency } \\
\text { before taking care of surgical } \\
\text { patients. }\end{array}$ & $\begin{array}{l}\text { Information gathered at } \\
\text { independent focus groups for } \\
\text { medical students, residents, and } \\
\text { faculty was used to develop the } \\
\text { curriculum. }\end{array}$ & $\begin{array}{l}\text { 1. Didactic lectures } \\
\text { 2. High-technology simulation } \\
\text { 3. Low-technology simulation } \\
\text { 4. Case-based learning }\end{array}$ & NS \\
\hline $\begin{array}{l}\text { Cohen et al, }{ }^{31} \\
2013\end{array}$ & $\begin{array}{l}\text { A short duration, high-intensity, } \\
\text { simulation-based course for } \\
\text { medical students designed to } \\
\text { improve confidence, enhance skills, } \\
\text { and ease the transition into } \\
\text { residency. }\end{array}$ & $\begin{array}{l}\text { Topics for the boot camp were } \\
\text { selected based on feedback from } \\
\text { historic control interns and } \\
\text { program faculty. }\end{array}$ & $\begin{array}{l}\text { 1. Small group learning } \\
\text { 2. Didactic lectures } \\
\text { 3. Low-technology simulation } \\
\text { 4. High-technology simulation } \\
\text { 5. E-learning }\end{array}$ & $\begin{array}{l}\text { Formative } \\
\text { feedback }\end{array}$ \\
\hline
\end{tabular}

Abbreviations: ACOS, American College of Surgeons; SMS, senior medical student; NS, not specified; PGY-1, postgraduate year-1.

\section{Knowledge Acquisition}

For improvements in knowledge acquisition, 4 boot camp studies provided pretest/posttest data on 5 outcome measures for trainees by mean scores in the form of multiple-choice and short answer question examinations (FIGURE 2). Studies included 5 different knowledge outcomes such as general and course-specific surgical knowledge, ${ }^{25}$ and knowledge of surgical ward management. ${ }^{29} \mathrm{~A}$ reported random-effects model for the combined effect sizes showed that participation in boot camps significantly improved learners' medical and surgical knowledge $(d=$ $2.08 ; 95 \%$ CI $1.20-2.96 ; P<.001)$. The heterogeneity was found to be high with an $I^{2}$ statistic value of $82.1 \%(P<$ $.01)$. Only 2 studies using comparison groups provided data on knowledge acquisition, and meta-analytic comparisons were not performed.

\section{Confidence in Clinical Abilities}

Eight studies provided pretest/posttest data for 37 outcomes related to improvement in trainees' perceived confidence, measured on Likert scales from strongly disagree to strongly agree (F I G U R E 3). For example, Todd et $\mathrm{al}^{28}$ surveyed (pretest/posttest) first-year residents about their confidence to manage a number of clinical presentations from sepsis, acute hypoxia, chest pain, decreased urine output, and postoperative fever. A random-effects model for the combined effect sizes showed significant improvement in confidence with a large effect size of $d=$
1.89 (95\% CI 1.63-2.15, $P<.001)$. The heterogeneity was found to be moderate with an $I^{2}$ statistic value of $65.4 \%$ ( $P$ $<.01$ ). Only 2 studies using comparison groups provided data on competence, and meta-analytic comparison was not performed.

\section{Discussion}

As shown by the number of studies that reported on the effectiveness of boot camps to enhance learners' transition to the next stage of their training, boot camps as a medical education strategy are still in their infancy. In identifying articles to include in our meta-analysis, we encountered a variety of definitions for boot camps. Although exact wording differed, certain commonalities were present: All studies described boot camps as courses/sessions aimed to prepare or orient learners undergoing clinical transitions, boot camps were structured as short and focused courses, and a wide variety of educational methods were applied in each camp with a particular focus on the use of simulation. In addition, most boot camps described a focus on providing immediate and/or formative feedback to participants in keeping with the Ericsson ${ }^{13}$ theory on how to support the development of expert performance.

Based on the boot camp descriptions from the literature included in the meta-analysis, the following definition is proposed: "A boot camp is a focused course designed to enhance learning, orientation, and preparation for learners entering a new clinical role. This is achieved through the 


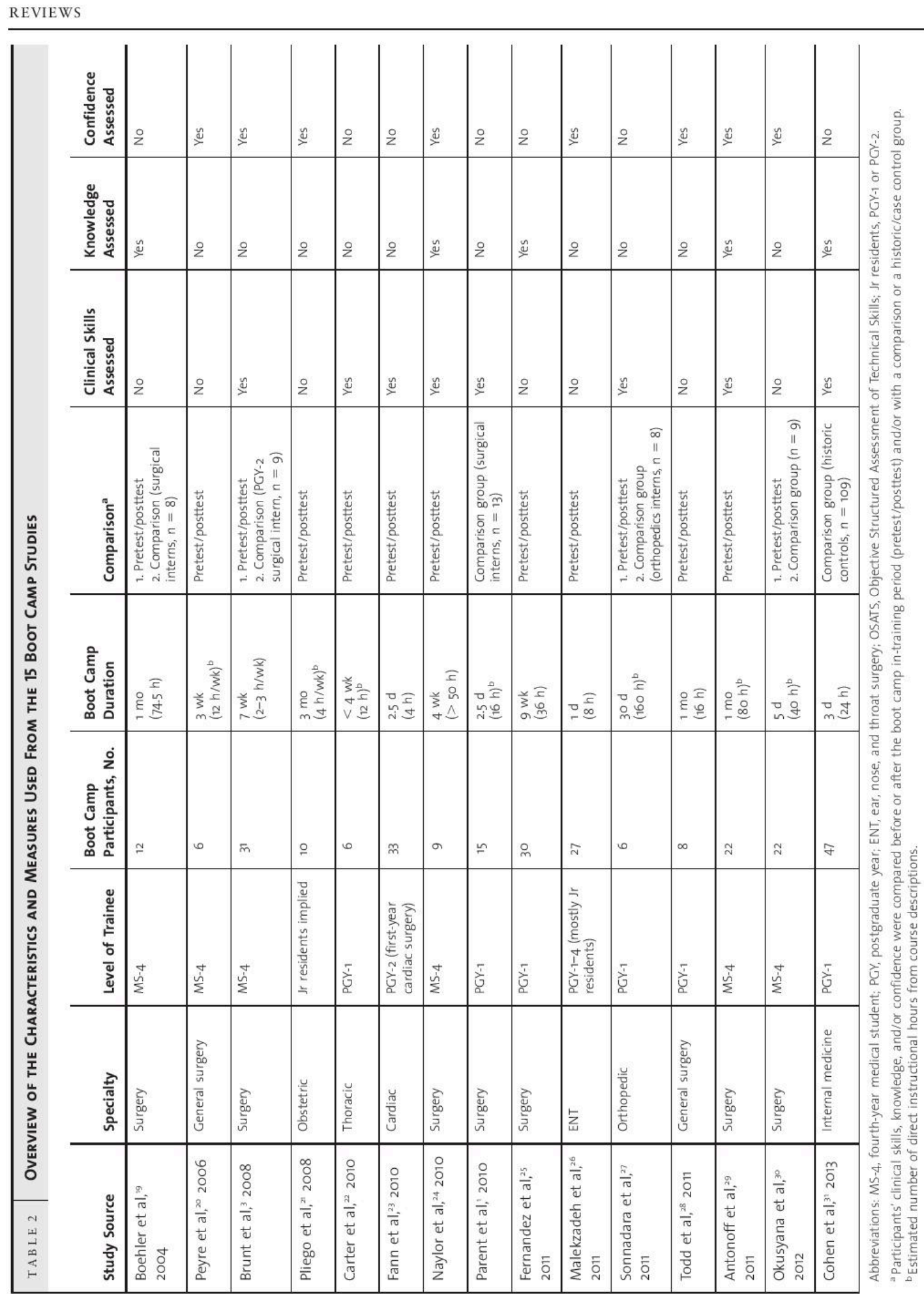

648 Journal of Graduate Medical Education, December 2014 


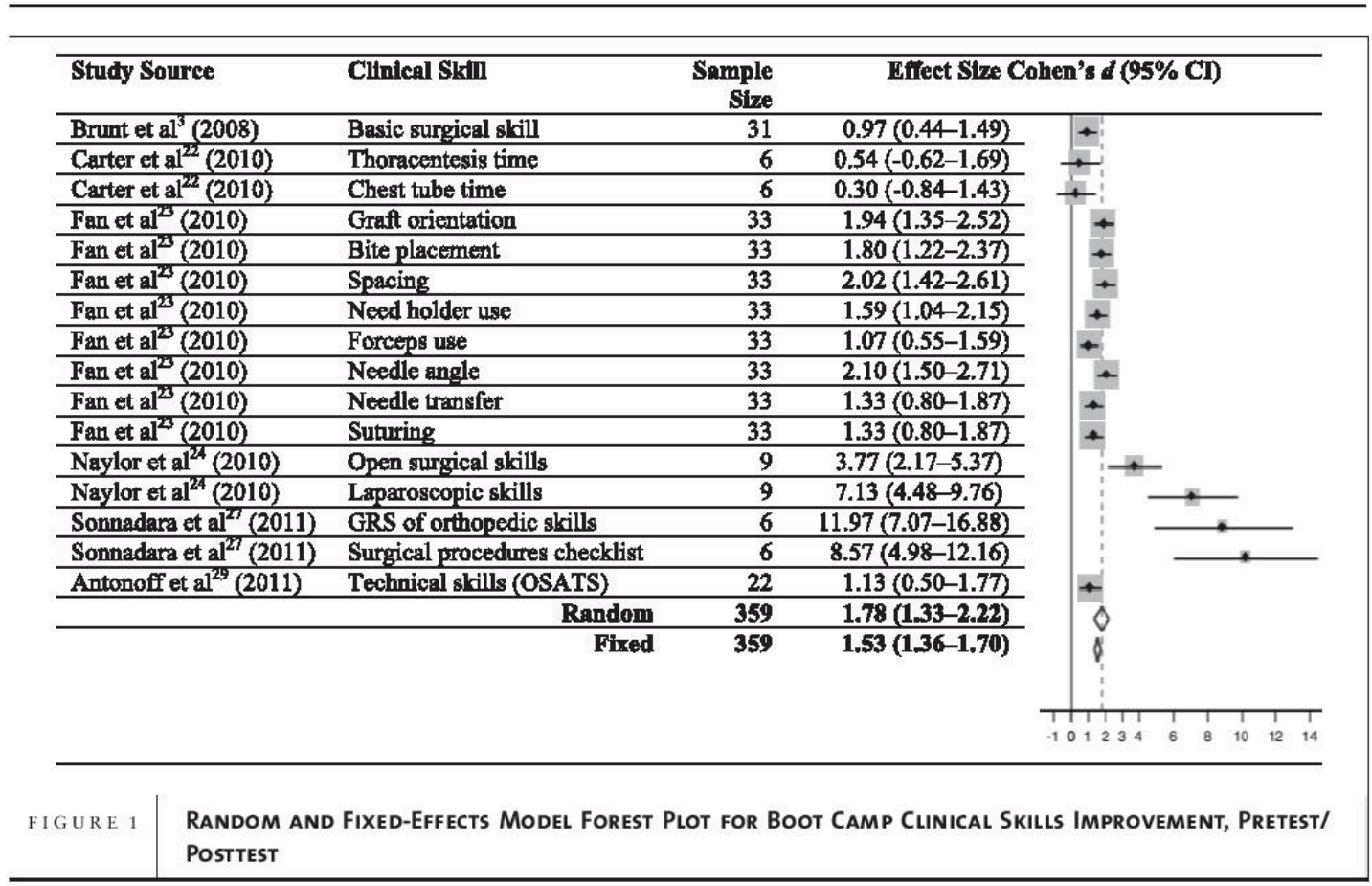

Abbreviations: GRS, global rating scale; OSATS, Objective Structured Assessment of Technical Skills.

use of multiple educational methods with a focus on deliberate practice with formative feedback." In support of the Ericcson's ${ }^{13}$ theory of what is required to develop expertise, the introduction of boot camp courses during career transition periods in clinical training may enhance trainees' clinical skills, knowledge, and confidence. The main finding of the meta-analysis is that learners who completed boot camps had significantly "large" improvements in (1) clinical skills development ( $d=1.78 ; 95 \%$ CI $1.33-2.22 ; P<.001),(2)$ knowledge acquisition $(d=2.08$;
95\% CI 1.20-2.96; $P<.001$ ), and (3) perceived confidence $(d=1.89 ; 95 \%$ CI $1.63-2.15 ; P<.001)$.

The effect sizes can also be interpreted as the average percentile standing of the trainees before and after participation in the boot camp course. ${ }^{15}$ An effect size of $d$ $=1.50$ indicates that the means of the posttest participants are at the 93.3 percentile for the means of their pretest scores or a nonboot camp (no intervention) comparison group. The boot camps studies included in this analysis demonstrated that an educational intervention that

\begin{tabular}{|c|c|c|c|c|c|}
\hline TABLE 3 & \multicolumn{5}{|c|}{ Random-Effects Model (Cohen's $d$ ) of the Clinical Skills, Knowledge, and Confidence Domains ${ }^{a}$} \\
\hline \multicolumn{2}{|c|}{ Boot Camp Domain Measure } & $\begin{array}{l}\text { Comparison } \\
\text { Analysis }\end{array}$ & $\begin{array}{l}\text { No. of Studies } \\
\text { (Outcomes) Included }\end{array}$ & $\begin{array}{l}\text { Boot Camp } \\
\text { Participants, No. }\end{array}$ & $\begin{array}{l}\text { Combined Effect Size } \\
\text { Cohen's } d(95 \% \mathrm{Cl})^{\mathrm{b}}\end{array}$ \\
\hline \multicolumn{2}{|c|}{ Clinical (and/or procedural) skills } & Pretest/posttest & $6(16)$ & 359 & $1.78(1.33-2.22)$ \\
\hline \multicolumn{2}{|c|}{ Clinical (and/or procedural) skills } & $\begin{array}{l}\text { Boot camp versus } \\
\text { comparison group }\end{array}$ & $4(9)$ & 207 & $2.73(1.36-4.07)$ \\
\hline \multicolumn{2}{|c|}{ Knowledge (medical or surgical) } & Pretest/posttest & $4(5)$ & 95 & $2.08(1.20-2.96)$ \\
\hline \multicolumn{2}{|l|}{ Confidence } & Pretest/posttest & $8(37)$ & 561 & $1.89(1.63-2.15)$ \\
\hline
\end{tabular}

${ }^{a}$ Based on data from 14 studies identified in a meta-analysis of the research on the effectiveness of boot camp programs to enhance clinical skills, knowledge,

and confidence during transition periods in clinical training.

${ }^{\mathrm{b}}$ Cohen's $d$ values $\geq 0.80$ indicate a large effect size change. 


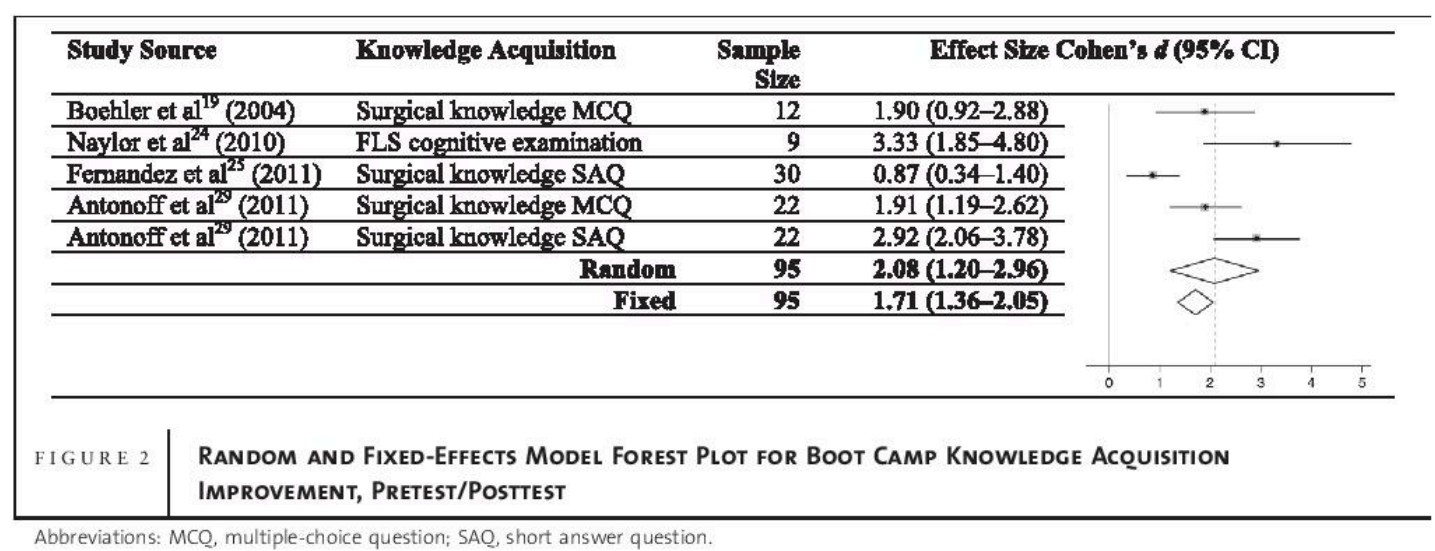

includes the use of deliberate practice in the development of clinical skills, knowledge, and confidence may provide immediate benefits to trainees.

To further support these findings, a meta-analysis of the combined effect sizes for clinical skill improvement across studies that used a comparison group demonstrated a large effect size difference between the intervention and comparison groups $(d=2.73 ; 95 \%$ CI $1.36-4.07 ; P<.001)$. Comparison groups used residents at similar levels of training, ${ }^{1,3,27,31}$ and the improvement found can be attributed to the course itself rather than to a maturation or testing effect. We were unable to perform a similar analysis for knowledge or confidence because of the few comparison group studies reporting these outcomes.

Although the combined effect sizes for each of the clinical skills, knowledge acquisition, and perceived confidence measures were all statistically significant, there is a significant amount of heterogeneity between the boot camp studies. ${ }^{18}$ The heterogeneity for studies that reported on clinical skills development was found to be moderately high $\left(I^{2}=81.2 \%, P<.01\right)$, reflecting variability in the skills being assessed (ie, tying a 2 handed knot, neonatal resuscitation, chest tube insertion), and in the assessment measures used (eg, length of checklists, types of global rating scales). Studies that reported on knowledge outcomes also had moderately high levels of heterogeneity $\left(I^{2}=82.1 \%, P<.01\right)$. This can be partly explained by the lack of standardization between examinations to assess the trainees' knowledge acquisition. Studies that reported on confidence improvement measures showed the lowest levels of heterogeneity $\left(I^{2}=65.4 \%, P<.01\right)$, which is explained in part by the fact that all confidence measures being assessed using similar self-report questionnaires that had trainees report on their confidence in their abilities as a result of participation in the boot camp.
The remainder of the heterogeneity among studies is to be expected because of less-rigorous research designs in these studies. For example, none of the studies in this metaanalysis used a random selection of trainees, and most used single group, pretest/posttest designs. Use of pretest and posttest scores can increase variability among studies, depending on when the examinations were given in relation to the end of boot camp (eg, posttest scores from immediately after the intervention can be influenced by the pretest in 1- to 3-day boot camp courses, whereas boot camps that were run for up to 4 to 7 weeks can be influenced by maturation effects). Although there was moderately high heterogeneity among studies (a reflection of the variability in the intensity and duration of the boot camp interventions), a fairly symmetric funnel plot analysis showed that publication bias in favor of positive findings only was not likely.

\section{Limitations}

We found significant variability among studies in the specialties involved, the number of participants (range from 6 to 47), the stage of the trainees' transition (eg, from medical school to residency or to a residency specialty), and the duration of the boot camp. Aside from heterogeneity associated with the single group, pretest/posttest research designs, further limitations to the study include the restricted scope of the types of boot camps (ie, all but 1 study involved surgical disciplines), the range of outcomes assessed, and the lack of long-term follow-up with trainees. Regarding outcomes assessed, the majority reported in the studies represented a focus on medical expert-related competencies. This limits the extrapolation of the results to other clinical competencies, such as communication with patients and their families or the development of skills for collaborating with colleagues and coworkers; the main concern for trainees during transition to residency and 
REVIEWS

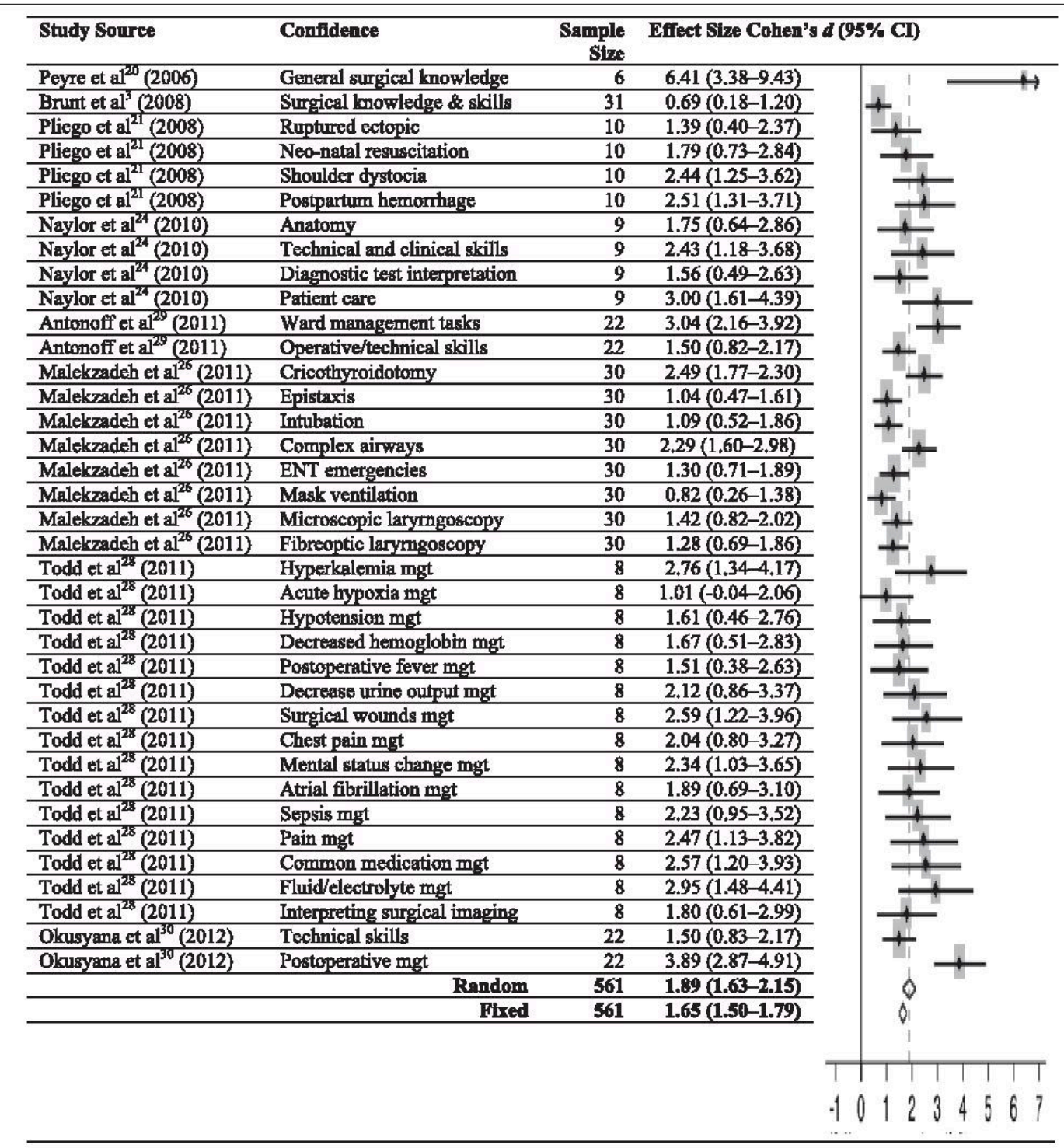

figure 3 Random and Fixed-Effects Model Forest Plot for Boot Camp Confidence Improvement, Pretest/ POSTTEST

Abbreviations: ENT, ear, nose, and throat; mgt, management.

fellowship appears to be development of surgical/procedural skills, including performing procedures, evaluating patients, and running codes. ${ }^{5,6}$ Finally, the results of the boot camp courses were limited because most studies provided little information about the costs and feasibility of running those camps or long-term follow-up data on retention of knowledge, skills, or attitudes.
As training hours become limited by widespread implementation of duty hour limits and as associated concerns for safety of care in teaching settings have become a public concern, there is a compelling need for curricular change and programs need to look for new ways to address those concerns. The use of boot camp courses may provide benefit during career transitions by increasing trainees' 
knowledge, skills, and confidence. Future studies that use rigorous research designs, such as comparison of boot camp intervention to other approaches, studies that include assessments of feasibility and costs, and research that explores delayed outcomes is needed for a comprehensive assessment of the benefits of this intervention.

\section{References}

1 Parent RJ, Plerhoples TA, Long EE, Zimmer DM, Teshome M, Mohr C, et al. Early, intermediate, and late effects of a surgical skills "boot camp" on an objective structured assessment of technical skills: randomized controlled study. J Am Coll Surg. 2010;210(6):984-989.

2 Nakayama DK, Steiber A. Surgery interns' experience with surgical procedures as medical students. Am J Surg. 1990;159(3):341-344.

3 Brunt LM, Halpin VJ, Klingensmith ME, Tiemann D, Matthews BD, Spitler JA, et al. Accelerated skills preparation and assessment for senior medical students entering surgical internship. J Am Coll surg. 2008;206(5):897-907.

4 Esterl RM Jr, Henzi DL, Cohn SM. Senior medical student "boot camp": can result in increased self-confidence before starting surgery internships. Curr Surg. 2006;63(4):264-268.

5 Nishisaki A, Hales R, Biagas K, Cheifetz I, Corriveau C, Garber N, et al. A multi-institutional high-fidelity simulation "boot camp" orientation and training program for first year pediatric critical care fellows. Ped Crit Care Med. 2009:10(2):157-162.

6 Jellinek MS, Todres ID, Catlin EA, Cassem EH, Salzman A. Pediatric intensive care training: confronting the dark side. Crit Care Med. 1993:21(5):775-779.

7 Bruce CT, Thomas PS, Yates BJ. Burnout and psychiatric morbidity in new medical graduates. Med J Aust. 2005:182(11):599.

8 van Walraven C, Jennings A, Wong J, Forster AJ. Influence of house-staff experience on teaching-hospital mortality: the "July phenomenon" revisited. J Hosp Med. 2011;6(7):389-394.

9 Inaba K, Recinos G, Teixeira PG, Barmparas G, Talving P, Salim A, et al. Complications and death at the start of the new academic year: is there a July phenomenon? J Trauma. 2010;68(1):19-22.

10 Poulose BK, Ray WA, Arbogast PG, Needleman J, Buerhaus PI, Griffin MR, et al. Resident work hour limits and patient safety. Ann Surg. $2005 ; 241(6): 847-856$

1 Sonnadara RR, Garbedian S, Safir O, Nousiainen M, Alman B, Ferguson P, et al. Orthopaedic Boot Camp II: examining the retention rates of an intensive surgical skills course. Surgery. 2012;151(6):803-807.

12 lobst WF, Sherbino J, Cate OT, Richardson DL, Dath D, Swing SR, et al. Competency-based medical education in postgraduate medical education. Med Teach. 2010;32(8):651-656.

13 Ericsson KA. Deliberate practice and acquisition of expert performance: a general overview. Acad Emerg Med. 2008;15(11):988-994.

14 McGaghie WC, Issenberg SB, Cohen ER, Barsuk JH, Wayne DB. Does simulation-based medical education with deliberate practice yield better results than traditional clinical education? A meta-analytic comparative review of the evidence. Acad Med. 2011;86(6):706-711.
15 Cohen J. Statistical Power Analysis for the Behavioral Sciences. 2nd ed. Hillsdale, NJ: Erlbaum Associates; 1988.

16 Wilson DB LM. Practical Meta-Analysis. 2nd ed. Thousand Oaks, CA: Sage Publications; 2001

17 DerSimonian R, Laird N. Meta-analysis in clinical trials. Control Clin Trials. $1986 ; 7(3): 177-188$.

18 Higgins JP, Thompson SG, Deeks JJ, Altman DG. Measuring inconsistency in meta-analyses. BMJ. 2003:327(7414):557-56o.

19 Boehler ML, Rogers DA, Schwind C, Fortune J, Ketchum J, Dunnington G. A senior elective designed to prepare medical students for surgical residency. Am J Surg. 2004:187(6):695-697.

20 Peyre SE, Peyre CG, Sullivan ME, Towfigh S. A surgical skills elective can improve student confidence prior to internship. I Surg Res. 2006:133(1):11-15

21 Pliego JF, Wehbe-Janek H, Rajab MH, Browning JL, Fothergill RE. OB/GYN boot camp using high-fidelity human simulators: enhancing residents' perceived competency, confidence in taking a leadership role, and stres hardiness. Simul Healthc. 2008;3(2):82-89.

22 Carter YM, Wilson BM, Hall E, Marshall MB. Multipurpose simulator for technical skill development in thoracic surgery. I Surg Res. 2010;163(2):186-191.

23 Fann Jl, Calhoon JH, Carpenter AJ, Merrill WH, Brown JW, Poston RS, et al. Simulation in coronary artery anastomosis early in cardiothoracic surgical residency training: the Boot Camp experience. J Thorac Cardiovasc Surg. 2010;139(5):1275-1281.

24 Naylor RA, Hollett LA, Castellvi A, Valentine RJ, Scott DJ. Preparing medical students to enter surgery residencies. Am J Surg. 2010;199(1):105-109.

25 Fernandez GL, Page DW, Coe NP, Lee PC, Patterson LA, Skylizard L, et al. Boot camp: educational outcomes after 4 successive years of preparatory simulation-based training at onset of internship. I Surg Educ. 2012;69(2):242-248.

26 Malekzadeh S, Malloy KM, Chu EE, Tompkins J, Battista A, Deutsch ES. ORL emergencies boot camp: using simulation to onboard residents. Laryngoscope. 2011;121(10):2114-2121.

27 Sonnadara RR, Van Vliet A, Safir O, Alman B, Ferguson P, Kraemer W, et al. Orthopedic boot camp: examining the effectiveness of an intensive surgical skills course. Surgery. 2011,149(6):745-749.

28 Todd SR, Fahy BN, Paukert J, Johnson ML, Bass BL. Surgical intern survival skills curriculum as an intern: does it help? Am J Surg. 2011;202(6):713-719.

29 Antonoff $M B$, D'Cunha J. PGY-1 surgery preparatory course design: Identification of key curricular components. I Surg Educ. 2011;68(6): $478-484$.

30 Okusanya OT, Kornfield ZN, Reinke CE, Morris JB, Sarani B, Williams NN, et al. The effect and durability of a pregraduation boot camp on the confidence of senior medical student entering surgical residencies. J surg Educ. 2012;69(4):536-543

31 Cohen ER, Barsuk JH, Moazed F, Caprio T, Didwania A, McGaghie WC, et al. Making July safer: simulation-based mastery learning during intern boot camp. Acad Med. 2013:88(2):233-239.

32 Martin JA, Regehr G, Reznick R, MacRae H, Murnaghan J, Hutchison C, et al. Objective structured assessment of technical skill (OSATS) for surgical residents. Br J Surg. 1997;84(2):273-278. 
DA UNIVERITY OF

⿶凵⿻尸丨 AIBERTA

\section{Use of JGME article}

1 message

Journal of Graduate Medical Education

Dear Dr. Blackmore,

Thank you for your email.

JGME grants permission for you to use your article for your final thesis.

Please let us know if you have any questions.

Kind Regards,

Amanda Plashal

Editorial Associate

Journal of Graduate Medical Education 
APPENDIX B: NEEDS ASSESSMENT SURVEY MANUSCRIPT WITH PERMISSION FOR USE FROM JOURNAL 


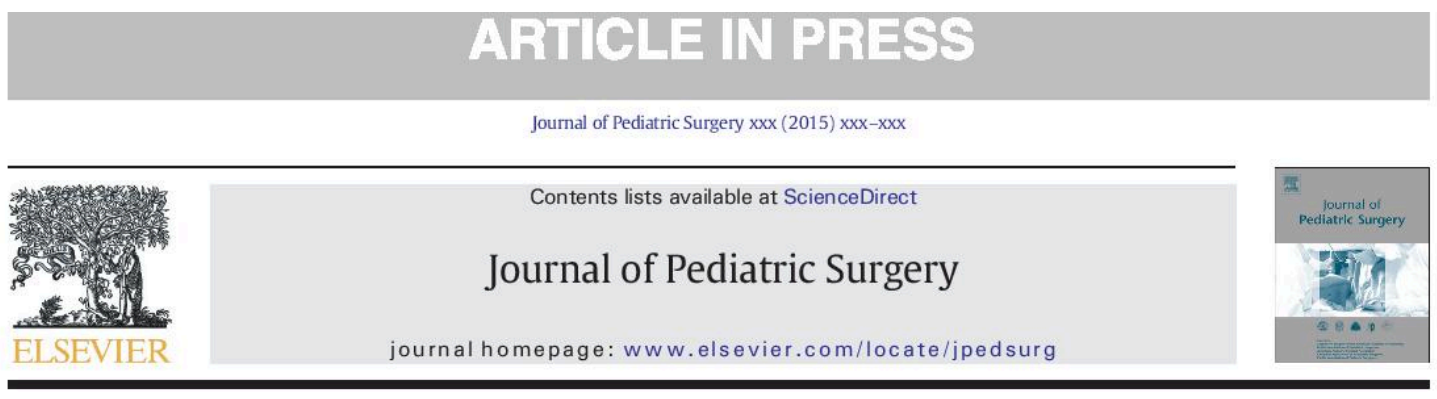

\title{
Targeted needs assessment for a transitional "boot camp" curriculum for pediatric surgery residents
}

\author{
Christopher Blackmore ${ }^{\mathrm{a}}$, Steve Lopushinsky ${ }^{\mathrm{a}, \mathrm{b}}$, Jocelyn Lockyer ${ }^{\mathrm{c}}$, Elizabeth Oddone Paolucci ${ }^{\mathrm{a}, \mathrm{c}, *}$ \\ a Department of Surgery, University of Calgary, Calgary, Alberta, Canada \\ bection of Pediatric Surgery, University of Calgary, Calgary, Alberta, Canad \\ ' Department of Community Health Sciences, University of Calgary, Calgary, Alberta, Canada
}

\section{A R T I C L E I N F O}

\section{Article history:}

Received 31 January 2015

Accepted 13 February 2015

Available online $\mathrm{xxxx}$

\section{Key words:}

Boot camp

Competency-based

Medical education

Career transitions pediatric surgery

\begin{abstract}
A B S T R A C T
Introduction: Transition periods in medical education are associated with increased risk for learners and patients. For pediatric surgery residents, the transition to training is especially difficult as learners must adjust to new patient populations. In this study we perform a targeted needs assessment to determine the ideal content and format of a pediatric surgery boot camp to facilitate the transition to residency.

Methods: A needs assessment survey was developed and distributed to pediatric surgery residents and staff across North America. The survey asked participants to rank 30 pediatric surgical diagnoses, 20 skills, and 11 physiological topics on "frequency" and "importance". Items were then ranked using empirical methods. The survey also evaluated the preferred boot camp format.

Results: In total, 12 residents and 23 staff completed the survey. No significant differences were identified between staff and residents in survey responses. The top 5 topics identified for inclusion in a boot camp were: (1) fluid and electrolyte management, (2) appendicitis, (3) pediatric hernias, (4) nutrition and (5) pain management. The preferred format for a boot camp was 3-4 days in duration applying a blend of educational methods. Conclusions: Based on the results of the needs assessment survey, a novel pediatric surgery boot camp curriculum can be developed.

(c) 2015 Published by Elsevier Inc.
\end{abstract}

Medical education in North America consists of well-defined stages connected by critical transition periods as one progresses from medical school to clinical practice. Focusing on Post-Graduate Medical Education (PGME), multiple studies have cited the transition from medical school to surgical residency as being stressful because of the variability in surgical skills exposure during clerkship as well as the increased number of clinical responsibilities [1-4]. These transition periods have also been associated with higher rates of psychiatric morbidity and burnout among trainees [5].

Of concern to medical educators and the community at large, transition periods may introduce potential risk for patients. The "July Phenomenon" is a well-studied effect predicting increased patient morbidity and mortality at teaching hospitals at the start of the academic year; a time that parallels most major transitions in medical training. In support of this, recent studies have shown that there is an increased risk of errors accounting for preventable complications during July [6,7].

From a pediatric surgery perspective, the Canadian Pediatric Adverse Events Study has identified that more children experience adverse events in academic centers compared to community centers [8]. This discrepancy was most pronounced in surgical patients, where those treated at academic centers experienced higher rates of adverse events ( $37.2 \%$ of patients) compared to community centers (21.5\% of patients) [8].

http://dx.doi.org/10.1016/j.jpedsurg.2015.02.044

0022-3468/C 2015 Published by Flsevier Inc.
Given the above concerns, there has been a strong push toward new methods of teaching and evaluation in PGME [9]. Programs are beginning to adopt competency-based frameworks to ensure that individual learner's skills match their progress through the continuum of medical education. In Canada, this is exemplified by the Royal College of Physicians and Surgeons of Canada (RCPSC) Competence by Design initiative [10]. In the United States, the Accreditation Council for Graduate Medical Education (ACGME) is implementing the Next Accreditation System (NAS) based on competency milestones [11]. In addition, many surgical programs have adopted "boot camps" to train residents during the critical transition to residency [12-15].

Pediatric surgery is unique in that trainees obtain their initial specialization in adult general surgery before transitioning to pediatrics. At the start, trainees have varying degrees of exposure to pediatrics and varying comfort levels in addressing pediatric issues. During informal discussions with pediatric surgery residents and staff, the transition into pediatric surgery has been described as particularly difficult. As a result, it was timely to investigate the perceptions of residents and staff regarding the need to develop an educational intervention for new pediatric surgery residents.

The purpose of this study was to conduct a targeted needs assessment of key stakeholders (i.e., residents and staff) in order to: (1) identify differences in the perceived needs for a pediatric surgery boot camp among stakeholders; (2) assess empirical ranking methods to inform the development of the future curriculum; (3) identify which pediatric

Please cite this article as: Blackmore C, et al, Targeted needs assessment for a transitional "boot camp" curriculum for pediatric surgery residents, J Pediatr Surg (2015), http://dx.doi.org/10.1016/j.jpedsurg.2015.02.044 


\section{ARTICLE IN PRIESS}

surgical diagnoses, skills, and physiological topics should be included as curricular content in a pediatric surgery boot camp for new residents; and (4) determine the preferred boot camp duration, structure and educational methods to be employed. To the best of our knowledge, this study was the first of its kind to gather the information necessary to inform the development of a pediatric surgery boot camp curriculum.

\section{Methods}

\subsection{Participants}

Participants comprised the entire population of the Canadian Associa tion of Pediatric Surgeons (CAPS; $n=172$ ). Additionally, Canadian and American trainees were targeted via their program directors who were asked to forward the needs assessment survey to their current residents. Although exact numbers could not be determined, the survey was estimated to have been distributed to approximately 200 participants.

\subsection{Targeted needs assessment: survey development}

In consultation with pediatric surgery staff, residents and a curricular design expert (J.L) at the University of Calgary, a targeted needs as sessment survey was developed. The survey consisted of two parts: (1) boot camp content, and (2) boot camp format. For the content section, three overarching domains were identified: (1) pediatric surgica diagnoses; (2) pediatric surgical skills; and (3) pediatric physiology. To ensure content validity, each of the pediatric surgical diagnoses $(n=30)$, skills $(n=20)$, and physiology topics $(n=11)$ included in the was were derived directly from the Royal College of Physician and Surgeons of Canada Objectives in Training for Pediatric Surgery Medical Expert competencies [16]. These objectives closely mirror those outlined by the Accreditation Council for Graduate Medical Education Pediatric Surgery Milestone Project [17]

Participants were asked to rate each of the pediatric surgical diagnoses, skills, and physiology topics on the level of "importance" to new pediatric surgery residents during their transition to training, and the "frequency" with which these items were encountered by pediatric surgeons or residents. This was done using a 5-point Likert scale. For importance, the scale ranged from " 1 " referring to "Not at all Important" to a score of " 5 " referring to "Very Important." For frequency, the scale ranged from " 1 " referring to "Less than Once per Year" to " 5 " referring to "More than Once per Week."

Our rating system was adapted from a similar study designed to create a rank order list of topics for inclusion in an internal medicine examination blueprint [18]. The "importance" score was designed to incorporate topics deemed critical for new trainees based on thei level of urgency and room for error. The "frequency" score aimed to capture topics encountered most often by a new trainee. By combining these factors as described below, we aimed to create a rank order list of topics deemed most critical for a pediatric surgical boot camp. This list would be used to develop goals, objectives and ultimately a pediatric surgery transitional "boot camp" curricular outline.

The boot camp format section of the survey involved multiple choice. short answer, and open-ended questions to determine the preferre format of the boot camp. Specifically, questions regarding the perceived ideal duration, instructional methods, and structure were posed. Participants were also asked to describe other competencies and content areas they felt should be included in a pediatric surgery boot camp. The survey was created using SurveyMonkey (SurveyMonkey Inc., Palo Alto, CA) and distributed via an emailed electronic link. Data were collected anonymously.

\subsection{Data analysis}

All statistical analyses were performed using SPSS version 22 (SPSS Inc., Chicago, IL). For the content part of the survey, the mean scores and standard deviations were calculated for importance and frequency ratings for all staff and resident respondents. For each domain (pediatric surgical diagnoses, skills and physiology), multivariate analysis of variance (MANOVA) was applied comparing staff and residents to identify any differences in how the key stakeholders perceived topics on the level of importance or frequency.

To determine which content should be included in a boot camp curriculum, we applied methodology described by Smith and Beran [19] for the development of rank order lists for clinical presentations. Given our smaller sample size, we were able to test four of the five methods for combining ratings of frequency and importance. The Rasch model was excluded from analysis given its requirement for large sample sizes [19]. The first method involved ranking based solely on "frequency" which determines how often the topic is encountered in practice. The second method involved ranking on "importance" ratings. The third method, the multiplicative model, involved multiplying the "frequency" and "importance" ratings to obtain a composite score, which provided equal weighting to both ratings. Lastly, the three-step model is a form of assigning weights on both frequency and severity. In this model, items ranked highly on both scales received higher weighting than items ranked highly on a single scale [19].

For the boot camp format section of the survey, answers were tabulated using frequencies. Open-ended questions were transcribed and themed. The University of Calgary Conjoint Health Research Ethics Board approved this study.

\section{Results}

\subsection{Participants}

In total, 12 residents and 23 pediatric surgery staff completed our survey, representing an estimated response rate of $18 \%(n \approx 200)$. Overall, $57 \%(n=20)$ of survey respondents were male, and $57 \%(n=20)$ had completed or were registered in training programs in Canada, $40 \%$ ( $n=$ 14) had completed or were registered in programs within the United States, and 3\% $(n=1)$ had completed training outside North America.

\subsection{Targeted needs assessment survey: boot camp content}

Mean ratings of each topic were tabulated for frequency and importance for residents and staff under each domain: pediatric surgical diagnoses (Table 1), pediatric surgical skills (Table 2 ), and pediatric physiology (Table 3). The MANOVAs comparing resident and staff ratings for importance, as well as frequency, revealed no significant differences between stakeholders (Tables 1-3).

\subsection{Rank order method comparison}

For the topics within each domain (pediatric surgical diagnoses, skills and physiology), the four ranking methods were applied and compared with Spearman's correlations $(\rho)$ in order to determine consistency among methods (Table 4). All four rankings methods were significantly correlated to one another. The frequency and importance methods had the lowest correlation $(\rho=0.59, p<0.01)$, while the three-step model and multiplicative model were the most highly correlated methods $(\rho=0.99, p<0.01)$.

\subsection{Targeted needs assessment: boot camp format}

Of the domains evaluated by our survey, participants ranked pediatric surgical diagnoses as most important, pediatric physiology as the second most important, and pediatric surgical skills as least important to focus on in a pediatric surgery boot camp.

The favored duration of the boot camp was three to four days ( $n=$ $12 ; 34 \%)$, followed by five to six days $(n=8 ; 23 \%)$, seven days or longer

Please cite this article as: Blackmore C, et al, Targeted needs assessment for a transitional "boot camp" curriculum for pediatric surgery residents, J Pediatr Surg (2015), http://dx.doi.org/10.1016/j.jpedsurg.2015.02.044 


\section{ARTICLE IN PRESS}

C. Blackmore et al. / Journal of Pediatric Surgery $x x x$ (2015) $x x x-x x x$

Table 1

Pediatric surgical diagnoses frequency and importance ratings by residents and staff ( $n=35$ ).

\begin{tabular}{|c|c|c|c|c|}
\hline \multirow[t]{2}{*}{ Pediatric surgical diagnoses } & \multicolumn{2}{|l|}{ Frequency data } & \multicolumn{2}{|l|}{ Importance data } \\
\hline & $\begin{array}{l}\text { Staff }(n=23), \\
\text { average (SD) }\end{array}$ & $\begin{array}{l}\text { Resident }(n=12) \text {, } \\
\text { average (SD) }\end{array}$ & $\begin{array}{l}\text { Staff }(n=23), \\
\text { average }(\mathrm{SD})\end{array}$ & $\begin{array}{l}\text { Resident ( } n=12) \text {, } \\
\text { average (SD) }\end{array}$ \\
\hline Appendicitis & $4.8(0.4)$ & $5.0(0.0)$ & $4.7(0.5)$ & $4.8(0.5)$ \\
\hline Pediatric hernias & $4.9(0.3)$ & $4.8(0.4)$ & $4.8(0.4)$ & $4.8(0.9)$ \\
\hline Pediatric burns and trauma & $4.2(0.9)$ & $4.4(0.9)$ & $4.3(1.0)$ & $4.6(0.5)$ \\
\hline Pyloric stenosis & $4.0(0.5)$ & $4.0(0.4)$ & $4.7(0.5)$ & $4.8(0.5)$ \\
\hline Necrotizing enterocolitis & $3.7(0.7)$ & $3.9(0.5)$ & $4.8(0.4)$ & $4.9(0.3)$ \\
\hline Neonatal bowel obstruction & $3.6(0.6)$ & $3.8(0.8)$ & $4.8(0.4)$ & $4.8(0.4)$ \\
\hline Short bowel syndrome & $3.4(0.7)$ & $4.0(0.6)$ & $4.3(0.8)$ & $4.3(0.8)$ \\
\hline Abdominal wall defects & $3.4(0.7)$ & $3.7(0.5)$ & $4.7(0.4)$ & $4.7(0.7)$ \\
\hline Intussusceptions & $3.6(0.5)$ & $3.3(0.9)$ & $4.7(0.6)$ & $4.4(0.5)$ \\
\hline Solid malignancies & $3.4(0.6)$ & $3.6(0.5)$ & $4.3(0.8)$ & $4.4(1.2)$ \\
\hline Esophageal atresia/trachea-esophageal fistulas & $3.1(0.5)$ & $3.3(0.7)$ & $4.6(0.7)$ & $4.8(0.9)$ \\
\hline Anorectal conditions and malformations & $3.2(0.5)$ & $3.2(0.9)$ & $4.6(0.7)$ & $4.7(1.2)$ \\
\hline Congenital diaphragmatic hernia & $3.0(0.7)$ & $3.3(0.6)$ & $4.5(0.7)$ & $4.8(0.6)$ \\
\hline Hirschsprungs disease & $3.1(0.5)$ & $3.3(0.8)$ & $4.7(0.5)$ & $4.7(0.9)$ \\
\hline Congenital and acquired lung lesions & $3.1(0.5)$ & $3.2(0.6)$ & $4.3(0.8)$ & $4.3(1.2)$ \\
\hline Acute scrotum & $3.0(0.9)$ & $3.0(0.9)$ & $4.4(0.7)$ & $4.4(0.5)$ \\
\hline Inflammatory bowel disease & $3.3(0.5)$ & $3.4(0.5)$ & $4.0(0.9)$ & $3.9(0.8)$ \\
\hline Gastrointestinal and airway foreign bodies & $3.2(1.0)$ & $2.9(1.4)$ & $3.8(1.1)$ & $4.2(0.9)$ \\
\hline Congenital/Acquired neck lesions & $3.6(0.7)$ & $3.3(0.7)$ & $3.7(1.2)$ & $3.7(1.1)$ \\
\hline Chest wall defects & $3.7(0.9)$ & $3.1(0.7)$ & $3.3(1.1)$ & $3.7(1.2)$ \\
\hline Congenital heart anomalies & $3.7(1.1)$ & $3.9(1.6)$ & $3.0(1.0)$ & $3.3(1.4)$ \\
\hline Pediatric gastrointestinal bleeding & $3.0(0.8)$ & $3.1(0.8)$ & $3.9(0.9)$ & $3.8(0.8)$ \\
\hline Pediatric obesity & $3.4(1.3)$ & $3.8(1.2)$ & $2.9(1.1)$ & $3.3(1.0)$ \\
\hline Genitourinary conditions & $3.5(1.0)$ & $3.1(1.4)$ & $3.3(1.1)$ & $3.3(1.2)$ \\
\hline Hematologic malignancies & $3.6(0.8)$ & $3.7(0.7)$ & $3.3(0.9)$ & $3.0(1.0)$ \\
\hline Congenital hepatobiliary abnormalities & $2.6(0.7)$ & $2.4(0.9)$ & $4.2(0.8)$ & $4.2(1.2)$ \\
\hline Gynecologic conditions & $3.0(0.7)$ & $2.8(1.1)$ & $3.4(0.8)$ & $3.2(1.2)$ \\
\hline Splenic conditions (spherocytosis) & $2.6(0.5)$ & $2.5(0.8)$ & $3.4(0.9)$ & $3.2(1.3)$ \\
\hline Necrotizing soft tissue infections & $1.6(0.8)$ & $1.4(0.7)$ & $3.4(1.3)$ & $3.4(1.0)$ \\
\hline Intersex abnormalities & $1.6(0.8)$ & $1.8(1.0)$ & $3.0(1.1)$ & $3.1(1.4)$ \\
\hline MANOVA & $p=0.71 ; \mathrm{NS}$ & & $p=0.39 ; \mathrm{NS}$ & \\
\hline
\end{tabular}

NS $=$ not significant.

( $n=7 ; 20 \%$ ), and lastly one to two days $(n=5 ; 14 \%)$. A small group of survey respondents were undecided $(n=3 ; 9 \%)$.

To evaluate preferred educational methods, participants were asked to choose from a list one or more methods that were best suited to teach each domain. For pediatric surgical diagnoses, the top three educational methods identified in the survey were problem based learning ( $n=29$;
$83 \%$ ), e-Learning ( $n=26 ; 74 \%$ ) and small group teaching ( $n=24 ; 69 \%)$. For pediatric surgical skills, the top three methods were high fidelity simulation $(n=26 ; 74 \%)$, animal labs $(n=23 ; 66 \%)$ and low fidelity simulation ( $n=20,57 \%$ ). Lastly, for pediatric physiology, the top three educational methods were problem based learning ( $n=26$; $74 \%)$, e-Learning $(n=25 ; 71 \%)$ and didactic lectures $(n=24 ; 69 \%)$.

Table 2

Pediatric surgical skills frequency and importance ratings by residents and staff $(n=35)$.

\begin{tabular}{|c|c|c|c|c|}
\hline \multirow[t]{2}{*}{ Pediatric surgical skills } & \multicolumn{2}{|l|}{ Frequency data } & \multicolumn{2}{|l|}{ Importance data } \\
\hline & Staff $(n=23)$, average (SD) & Resident ( $n=12$ ), average (SD) & Staff $(n=23)$, average (SD) & Resident $(n=12)$, average (SD) \\
\hline Vascular access & $4.6(0.5)$ & $4.3(1.0)$ & $4.6(0.5)$ & $4.8(0.6)$ \\
\hline Advanced laparoscopy & $4.3(0.9)$ & $4.8(0.5)$ & $4.2(0.9)$ & $4.9(0.3)$ \\
\hline Advanced trauma life support & $3.8(0.9)$ & $4.3(0.6)$ & $4.7(0.5)$ & $4.7(0.5)$ \\
\hline Chest tube insertion & $3.8(0.7)$ & $3.5(0.7)$ & $4.5(0.7)$ & $4.4(0.7)$ \\
\hline Advanced thoracoscopy & $3.4(0.9)$ & $3.8(0.5)$ & $4.0(1.0)$ & $4.8(0.5)$ \\
\hline Clinical and radiologic clearance of C-spine & $3.8(1.1)$ & $4.1(0.8)$ & $3.9(1.0)$ & $3.8(1.1)$ \\
\hline Pediatric advanced life support (skills) & $3.1(1.1)$ & $3.3(0.8)$ & $4.0(1.0)$ & $4.3(0.9)$ \\
\hline Upper endoscopy & $3.3(1.2)$ & $3.2(1.0)$ & $3.6(1.1)$ & $3.7(1.1)$ \\
\hline Ultrasound guided procedures & $3.1(1.3)$ & $2.9(1.2)$ & $3.4(1.2)$ & $4.2(0.6)$ \\
\hline Trauma laparotomy & $2.2(0.9)$ & $2.4(0.8)$ & $4.2(0.7)$ & $4.5(0.7)$ \\
\hline Bronchoscopy & $2.8(0.8)$ & $2.6(1.2)$ & $3.5(1.0)$ & $3.7(0.9)$ \\
\hline Dialysis catheter insertion & $2.9(0.9)$ & $2.8(1.1)$ & $3.4(1.0)$ & $3.3(1.3)$ \\
\hline Sigmoidoscopy & $2.9(1.1)$ & $2.6(1.1)$ & $3.4(1.0)$ & $3.3(1.3)$ \\
\hline FAST & $2.7(1.4)$ & $3.0(1.4)$ & $3.4(1.1)$ & $3.0(1.5)$ \\
\hline Endotracheal intubation & $2.9(1.6)$ & $2.4(1.2)$ & $3.4(1.2)$ & $3.0(1.2)$ \\
\hline EQS cannulation & $2.2(1.3)$ & $2.5(1.2)$ & $3.0(1.2)$ & $4.2(1.1)$ \\
\hline Tracheostomy & $1.9(1.2)$ & $2.0(1.1)$ & $3.0(1.1)$ & $3.6(1.2)$ \\
\hline Intraosseous needle insertion & $1.8(0.9)$ & $1.8(1.1)$ & $3.5(1.0)$ & $3.2(1.2)$ \\
\hline Fasciotomy & $1.5(0.7)$ & $1.7(1.0)$ & $3.0(1.0)$ & $3.4(1.0)$ \\
\hline Emergency department thoracotomy & $1.1(0.3)$ & $1.3(0.5)$ & $2.7(1.4)$ & $3.3(1.2)$ \\
\hline MANOVA & $p=0.98 ; \mathrm{NS}$ & & $p=0.50 ; \mathrm{NS}$ & \\
\hline
\end{tabular}

Please cite this article as: Blackmore C, et al, Targeted needs assessment for a transitional "boot camp" curriculum for pediatric surgery residents, J Pediatr Surg (2015), http://dx.doi.org/10.1016/j.jpedsurg.2015.02.044 


\begin{tabular}{|c|c|c|c|c|}
\hline \multicolumn{5}{|c|}{ 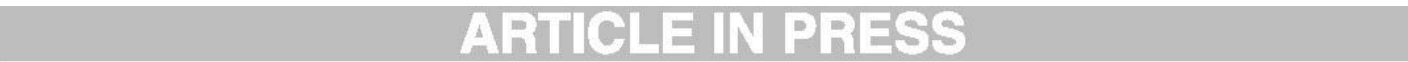 } \\
\hline \multicolumn{5}{|c|}{ C. Blackmore et al. / Journal of Pediatric Surgery $x x x$ (2015) $x x x-x x x$} \\
\hline \multicolumn{5}{|l|}{ Table 3} \\
\hline \multicolumn{5}{|c|}{ Pediatric physiology topic frequency and importance ratings by residents and staff $(n=35)$. } \\
\hline \multirow[t]{2}{*}{ Pediatric physiology topics } & \multicolumn{2}{|l|}{ Frequency data } & \multicolumn{2}{|l|}{ Importance data } \\
\hline & Staff $(n=23)$, average (SD) & Resident $(n=12)$, average (SD) & Staff $(n=23)$, average (SD) & $\overline{\text { Resident }(n=12) \text {, average (SD) }}$ \\
\hline Fluid and electrolyte management & $4.8(0.4)$ & $5.0(0.00)$ & $4.8(0.5)$ & $4.8(0.4)$ \\
\hline Nutrition & $4.7(0.6)$ & $4.6(1.2)$ & $4.5(0.6)$ & $4.8(0.5)$ \\
\hline Pediatric pain & $5.0(0.0)$ & $4.6(1.2)$ & $4.5(0.6)$ & $3.9(1.2)$ \\
\hline Gastrointestinal physiology & $4.3(0.7)$ & $4.6(0.5)$ & $4.0(0.9)$ & $4.2(0.8)$ \\
\hline Thermoregulation & $4.5(0.9)$ & $4.1(0.7)$ & $4.2(0.9)$ & $3.6(0.9)$ \\
\hline Shock & $3.6(0.8)$ & $3.9(0.5)$ & $4.7(0.4)$ & $4.7(0.5)$ \\
\hline Transfusion therapy and coagulation & $4.0(0.9)$ & $4.1(0.7)$ & $4.1(0.8)$ & $3.9(0.9)$ \\
\hline Pulmonary physiology & $3.7(0.8)$ & $4.2(0.7)$ & $4.0(1.0)$ & $4.2(0.7)$ \\
\hline Cardiac physiology & $3.5(.9)$ & $3.7(1.1)$ & $3.8(0.9)$ & $3.8(1.0)$ \\
\hline Pediatric advanced life support (physiology) & $2.7(1.3)$ & $3.1(0.9)$ & $4.1(1.0)$ & $4.0(1.0)$ \\
\hline Extracorporeal life support & $2.5(1.1)$ & $2.9(1.0)$ & $3.4(1.0)$ & $4.4(0.9)$ \\
\hline MANOVA & $p=0.54 ; \mathrm{NS}$ & & $p=0.70 ; \mathrm{NS}$ & \\
\hline
\end{tabular}

NS $=$ not significant.

In the open-ended section of the survey, participants were asked if there were additional competencies that should be included in a pediatric surgery boot camp. The most common theme identified by participants ( $n=10 ; 29 \%$ ) was to include a component on communication. This included communication with pediatric patients, difficult or anxious parents, surgical staff and pediatric colleagues. Other areas mentioned included pediatric drug dosing, recognizing pediatric abuse, and patient advocacy.

\section{Discussion}

This study consisted of developing and distributing a targeted needs assessment survey to key stakeholders in the field of pediatric surgery. Participants were surveyed regarding the content and format of a pediatric surgery boot camp. No significant differences were identified in survey responses between residents and staff indicating that all stakeholders, regardless of level of training, had similar perceptions as to which topics were critical for new trainees. Given these findings, the data were combined for residents and staff on all further analyses, thereby producing a total of 35 survey respondents.

In addition, four separate methods were evaluated for ranking topics to guide curricular content. While there were statistically significant correlations between all methods, the multiplicative and three-step models showed the most precise rankings, demonstrating a high correlation between both frequency and importance. These models demonstrated that participants tended to rank topics more consistently when both importance and frequency were taken into account, as opposed to independent consideration. In the analysis by Smith and Beran [19], the Rasch model was concluded to be the ideal method, although it was found to be highly correlated $(0.94, p<0.001)$ with the multiplicative model. Given Rasch modeling's requisite of large sample sizes and special software for its application, these factors may serve as a deterrent and limit its utility in the setting of curricular design. With this in mind, the multiplicative model was chosen as our preferred method given its simplicity in application compared to the more cumbersome three-step model or the Rasch model.

Applying the multiplicative model to the data from the content section of the survey, we identified the following as the top three ranked pediatric surgical diagnoses: (1) appendicitis, (2) pediatric hernias, and (3) pediatric burns and trauma. The top three pediatric surgical skills were: (1) vascular access, (2) advanced laparoscopy, and (3) advanced trauma life support. Lastly, the top three pediatric physiology topics were: (1) fluid and electrolyte management, (2) nutrition, and (3) pediatric pain management. These lists represent topics felt by stakeholders to be critical for new pediatric surgery residents to be familiar with because of the high likelihood that they will encounter them during their initial transition into the program, as well as the importance for residents to have a understanding of the topic given factors like low room for error or urgency in which it must be dealt with.

The format section of the survey identified that three to four days was the preferred duration for a pediatric surgery boot camp by over a third of participants. However, 20\% of participants chose 7 days or longer as the ideal format, indicating a preference toward more longitudinal style curricula. Furthermore, participants felt that content was best addressed using a variety of educational methods including problem based learning, small group teaching, e-Learning, didactic lectures, and simulation. When structuring the curriculum, participants felt that the focus should be primarily on critical pediatric surgical diagnoses and pediatric physiology. Pediatric surgical skills on the other hand were rated as least important. This was mirrored in the rank order list in which 8 of the top 20 ranked topics were related to pediatric surgical diagnoses, 8 were related to pediatric physiology, and only 4 were related to surgical skills (Table 4). These findings are not surprising given the significant differences between adult diagnoses and physiology compared to the novel pediatric diagnoses and physiology new residents' face at the start of their training. Surgical skills on the other hand demonstrate significant overlap between adult and pediatric surgery, likely explaining why these were felt to be less important for new residents. Lastly, in addition to medical expert competencies, participants felt that a boot camp would benefit from including other competencies, specifically communication and health care advocacy.

Combining the boot camp format preferences, from a feasibility perspective, a Friday to Sunday weekend course at a central location would allow for pooling of resources between programs across the country with minimal absence from training for residents. Furthermore, learner and educator time and resource commitment could be enhanced by implementing a blended curriculum, involving an online e-Learning component followed by a focused course involving didactic lectures, problem and case based learning, as well as simulation. Ideally, this type of boot camp course would supplement longitudinal curricula, while focusing on orientation and evaluation during the transition to discipline for new residents.

The biggest limitation to our study was the low number of survey respondents. Given the small number of pediatric surgical specialists across North America, we were limited in the total population. Using an electronic survey for data collection may have further impacted this given our reliance on program directors for survey distribution. Nonetheless, our response rate was found to be comparable to and consistent with other studies using electronic surveys [20]. In spite of these shortcomings, the combination of resident and staff data enabled us to attain reasonable numbers for appropriate statistical analysis. Furthermore, generalizability of results was maintained through surveying residents and staff across North America.

Through our targeted needs assessment, we were able to determine the educational needs for new pediatric surgery residents, as well the desired format through which these needs can be addressed during the transition into residency. Additionally, we have demonstrated an easily reproducible method of developing rank order lists of topics for inclusion in novel curricula. Going forward, the data collected from our survey will be used to develop a new competence based pediatric surgery boot camp curriculum.

Please cite this article as: Blackmore C, et al, Targeted needs assessment for a transitional "boot camp" curriculum for pediatric surgery residents, J Pediatr Surg (2015), http://dx.doi.org/10.1016/j.jpedsurg.2015.02.044 


\section{ARTICLE IN PRESS}

C. Blackmore et al. / Journal of Pediatric Surgery $x x x$ (2015) $x x x-x x x$

Table 4

Rank order method comparison using combined data for residents and staff $(N=35)$.

\begin{tabular}{|c|c|c|c|c|c|}
\hline \multirow[t]{2}{*}{ Rank (as per multiplicative model) } & \multirow[t]{2}{*}{ Topic } & \multicolumn{4}{|c|}{ Rank order method } \\
\hline & & Frequency & Importance & Multiplicative model & Three-step model \\
\hline 1 & Fluid and electrolyte management & 4.9 & 4.8 & 23.5 & 312 \\
\hline 2 & Appendicitis & 4.9 & 4.8 & 23.2 & 315 \\
\hline 3 & Pediatric hernias & 4.9 & 4.8 & 23.2 & 309 \\
\hline 4 & Nutrition & 4.6 & 4.6 & 21.2 & 303 \\
\hline 5 & Pediatric pain & 4.9 & 4.3 & 21.1 & 292 \\
\hline 6 & Vascular access & 4.5 & 4.6 & 20.8 & 304 \\
\hline 7 & Advanced laparoscopy & 4.4 & 4.5 & 19.7 & 291 \\
\hline 8 & Pediatric burns and trauma & 4.3 & 4.6 & 19.5 & 283 \\
\hline 9 & Pyloric stenosis & 4 & 4.8 & 19.1 & 306 \\
\hline 10 & Necrotizing enterocolitis & 3.8 & 4.9 & 18.7 & 285 \\
\hline 11 & Advanced trauma life support & 4 & 4.7 & 18.5 & 288 \\
\hline 12 & Gastrointestinal physiology & 4.4 & 4.1 & 18 & 276 \\
\hline 13 & Neonatal bowel obstruction & 3.6 & 4.8 & 17.5 & 267 \\
\hline 14 & Thermoregulation & 4.4 & 4 & 17.5 & 268 \\
\hline 15 & Shock & 3.7 & 4.7 & 17.4 & 273 \\
\hline 16 & Chest tube insertion & 3.7 & 4.5 & 16.5 & 266 \\
\hline 17 & Abdominal wall defects & 3.5 & 4.7 & 16.4 & 256 \\
\hline 18 & Transfusion therapy and coagulation & 4.1 & 4 & 16.3 & 267 \\
\hline 19 & Pulmonary physiology & 3.9 & 4.1 & 15.9 & 254 \\
\hline 20 & Short bowel syndrome & 3.6 & 4.3 & 15.7 & 249 \\
\hline 21 & Intussusception & 3.5 & 4.4 & 15.4 & 261 \\
\hline 22 & Solid malignancies & 3.5 & 4.4 & 15.3 & 246 \\
\hline 23 & Esophageal atresia/trachea-esophageal fistulas & 3.2 & 4.8 & 15.2 & 225 \\
\hline 24 & Advanced thoracoscopy & 3.5 & 4.2 & 15 & 243 \\
\hline 25 & Clinical and radiologic clearance of C-spine & 3.9 & 3.9 & 15 & 230 \\
\hline 26 & Anorectal conditions and malformations & 3.2 & 4.7 & 14.9 & 222 \\
\hline 27 & Congenital diaphragmatic hernia & 3.1 & 4.8 & 14.9 & 216 \\
\hline 28 & Hirschsprungs disease & 3.2 & 4.7 & 14.8 & 223 \\
\hline 29 & Cardiac physiology & 3.6 & 3.8 & 13.7 & 239 \\
\hline 30 & Congenital and acquired lung lesions & 3.1 & 4.3 & 13.6 & 216 \\
\hline 31 & Acute scrotum & 3 & 4.4 & 13.4 & 217 \\
\hline 32 & Inflammatory bowel disease & 3.3 & 3.9 & 13.1 & 224 \\
\hline 33 & Gastrointestinal and airway foreign bodies & 3.1 & 4.2 & 13 & 208 \\
\hline 34 & Pediatric advanced life support (skills) & 3.2 & 4.1 & 13 & 209 \\
\hline 35 & Congenital/Acquired neck lesions & 3.5 & 3.7 & 12.8 & 210 \\
\hline 36 & Chest wall defects & 3.5 & 3.7 & 12.8 & 192 \\
\hline 37 & Congenital heart anomalies & 3.7 & 3.3 & 12.2 & 191 \\
\hline 38 & Upper endoscopy & 3.3 & 3.6 & 11.9 & 203 \\
\hline 39 & Pediatric gastrointestinal bleeding & 3 & 3.8 & 11.5 & 200 \\
\hline 40 & Pediatric obesity & 3.5 & 3.3 & 11.4 & 174 \\
\hline 41 & Pediatric advanced life support (physiology) & 2.8 & 4.1 & 11.4 & 186 \\
\hline 42 & Genitourinary conditions & 3.4 & 3.3 & 11.2 & 186 \\
\hline 43 & Ultrasound guided procedures & 3 & 3.7 & 11.1 & 189 \\
\hline 44 & Hematologic malignancies & 3.6 & 3 & 10.8 & 190 \\
\hline 45 & Congenital hepatobiliary abnormalities & 2.5 & 4.2 & 10.5 & 164 \\
\hline 46 & Extracorporeal life support (physiology) & 2.7 & 3.8 & 10.2 & 176 \\
\hline 47 & Trauma laparotomy & 2.3 & 4.3 & 9.9 & 147 \\
\hline 48 & Bronchoscopy & 2.7 & 3.6 & 9.8 & 163 \\
\hline 49 & Dialysis catheter insertion & 2.9 & 3.4 & 9.7 & 166 \\
\hline 50 & Sigmoidoscopy & 2.8 & 3.4 & 9.5 & 157 \\
\hline 51 & FAST & 2.8 & 3.3 & 9.3 & 158 \\
\hline 52 & Gynecologic conditions & 2.9 & 3.2 & 9.2 & 162 \\
\hline 53 & Endotracheal intubation & 2.7 & 3.3 & 8.9 & 153 \\
\hline 54 & Splenic conditions (spherocytosis) & 2.6 & 3.2 & 8.1 & 138 \\
\hline 55 & Extracorporeal life support cannulation & 2.3 & 3.4 & 7.9 & 147 \\
\hline 56 & Tracheostomy & 1.9 & 3.2 & 6.1 & 113 \\
\hline 57 & Intraosseous needle insertion & 1.8 & 3.4 & 6 & 101 \\
\hline 58 & Necrotizing soft tissue infections & 1.5 & 3.4 & 5.3 & 92 \\
\hline 59 & Intersex abnormalities & 1.7 & 3.1 & 5.1 & 95 \\
\hline 60 & Fasciotomy & 1.6 & 3.1 & 4.9 & 90 \\
\hline 61 & Emergency department thoracotomy & 1.2 & 2.9 & 3.4 & 69 \\
\hline
\end{tabular}

Note: The topics are listed in order as by the ranking from the multiplicative model from highest to lowest.

\section{Acknowledgments}

Thank you to the Department of Surgery, University of Calgary for providing financial support for this project through the Calgary Surgical Research and Development Fund.

\section{References}

[1] Nakayama DK, Steiber A. Surgery interns' experience with surgical procedures as medical students. Am J Surg 1990;159(3):341-3 [discussion 344].

[2] Brunt LM, Halpin VJ, Klingensmith ME, et al. Accelerated skills preparation and assessment for senior medical students entering surgical internship. J Am Coll Surg 2008:206(5):897-904 [904-7].

Please cite this article as: Blackmore C, et al, Targeted needs assessment for a transitional "boot camp" curriculum for pediatric surgery residents, J Pediatr Surg (2015), http://dx.doi.org/10.1016/j.jpedsurg.2015.02.044 


\section{ARTICLE IN PRESS}

[3] Esterl Jr RM, Henzi DL, Cohn SM. Senior medical student "boot camp" can result in increased self-confidence before starting surgery intemships. Curr Surg 2006;63(4):264-8. [4] Parent RJ, Plerhoples TA, Long EE, et al. Early, intermediate, and late effects of a surgical skills "boot camp" on an objective structured assessment of technical skills: a randomized controlled study.J Am Coll Surg 2010;210(6):984-9.

[5] Willcock SM, Daly MG, Tennant CC, Allard BJ. Burnout and psychiatric morbidity in new medical graduates. Med J Aust 2004;181(7):357-60.

[6] van Walraven C, Jennings A, WongJ, et al. Influence of house-staff experience on teachin hospital mortality: the "July phenomenon" revisited. J Hosp Med 2011;6(7):389-94.

[7] Inaba K, Recinos G, Teixeira PG, et al. Complications and death at the start of the new academic year: is there a July phenomenon? J Trauma 2010;68(1):19-22.

[8] Matlow AG, Baker GR, Flintoft VF, et al. Adverse events among children in Canadian hospitals: The Canadian Paediatric Adverse Events Study. CMAJ 2012;184(13):E709-18.

[9] Lockyer J, Silver l, Oswald A, et al. The continuum of medical education. Competence by design: reshaping Canadian medical education; 2014. p. 130-41 (http://www. royalcollege.ca/portal/page/portal/rc/common/documents/educational_initiatives/ rc_competency-by-design_ebook_e.pdf].

[10] Snell L, Frank JR, Stoneham G, et al. Competency-based medical education; 2014. p. 99-105 /http://www.royalcollege.ca/portal/page/portal/rc/common/documents/ educational_initiatives/rc_competency-by-design_ebook_e.pdf].

[11] Holmboe ES, Edgar L Next accreditation system: milestones. https://www.acgme. org/acgmeweb/tabid/430/ProgramandlinstitutionalAccreditation// NextAccreditationSystem/Milestones.aspx. [Updated 2014. Accessed 06/27, 2014]
[12] Sonnadara RR, Garbedian S, Safir O, et al. Orthopaedic boot camp II: examining the retention rates of an intensive surgical skills course. Surgery 2012;151(6): 803-7.

[13] Selden NR, Origitano TC, Burchiel KJ, et al. A national fundamentals curriculum for neurosurgery PGY1 residents: The 2010 Society of Neurological Surgeons Boo Camp Courses, Neurosurgery 2012;70(4):971-81

[14] Sonnadara RR, Van Vliet A, Safir O, et al. Orthopedic boot camp: examining the effectiveness of an intensive surgical skills course. Surgery 2011;149(6):745-9.

[15] Antonoff MB, Shelstad RC, Schmitz C, et al. A novel critical skills curriculum for surgical interns incorporating simulation training improves readiness for acute inpatient care. J Surg Educ 2009;66(5):248-54.

[16] The Royal College of Physicians and Surgeons of Canada. Objectives in training in the subspecialty of pediatric surgery. http://rcpsc.medical,org/residency/certification objectives/ped-gen-surgery_e.pdf. [Updated 2012. Accessed 11/25, 2012].

[17] The Accrediation Council for Graduate Medical Education. The Pediatric Surgery Milestone Project. https://www.acgme.org/acgmeweb/Portals/0/PDFs/Milestones/ PediatricSurgeryMilestones.pdf. [Updated February 2014. Accessed 08/26, 2014].

[18] McLaughlin K, Lemaire J, Coderre S. Creating a reliable and valid blueprint for the internal medicine clerkship evaluation. Med Teach 2005;27(6):544-7.

[19] Smith SD, Beran TN. A comparison of empirical ranking methods of frequency an severity ratings of clinical presentations. Eval Health Prof 2012;35(4):383-95.

[20] Kaplowitz MD, Hadlock TD, Levine R. A comparison of web and mail survey response rates. Public Opin Q 2004;68(1):94-101.

Please cite this article as: Blackmore C, et al, Targeted needs assessment for a transitional "boot camp" curriculum for pediatric surgery residents, J Pediatr Surg (2015), http://dx.doi.org/10.1016/j.jpedsurg.2015.02.044 


\section{Re: Question Regarding CAPS Manuscript}

1 message

\section{Sarah Jones}

I am the editor for the edition you have my permission to use it in your thesis. Good luck 》> Christopher Blackmore $\quad 01 / 19 / 1518: 28 \mathrm{PM} \gg>$

Hello Dr. Jones

I have recently had a manuscript presented at CAPS accepted for publication in a future issue of the Journal of Pediatric Surgery (please see acceptance email below).

I am currently completing a MSc. in Medical Education, and the data presented in this article is part my my masters project. For my thesis, I am completing a manuscript based thesis and was hoping to use this article as one of the chapters of my manuscript.

Do you happen to know who I can contact or how I can go about obtaining permission to use this article in my thesis?

Thank you very much for your time.

Chris Blackmore PGY-4

Department of General Surgery

University of Calgary 
APPENDIX C: Needs Assessment Survey 


\section{Development of a Surgical "Boot Camp" Curriculum for Pediatric Surgery}

\section{Section 1: Pediatric Medical and Surgical Diagnoses}

The following page contains a list of PEDIATRIC MEDICAL/SURGICAL DIAGNOSES derived from the objectives in training for Pediatric Surgery created by the Royal College of Physicians and Surgeons of Canada. These objectives closely mirror the objectives set forth by the American Council for Graduate Medical Education.

\section{Please rate the following PEDIATRIC MEDICAL/SURGICAL DIAGNOSES based on the} following crit eria:

1) The level of IMPORTANCE to new pediatric surgery fellows during their initial transiition to fellowship

2) The FREQUENCY that they are encountered by pediatric surgeons or fellows

3) Your own level of CONFIDENCE in dealing with the topic

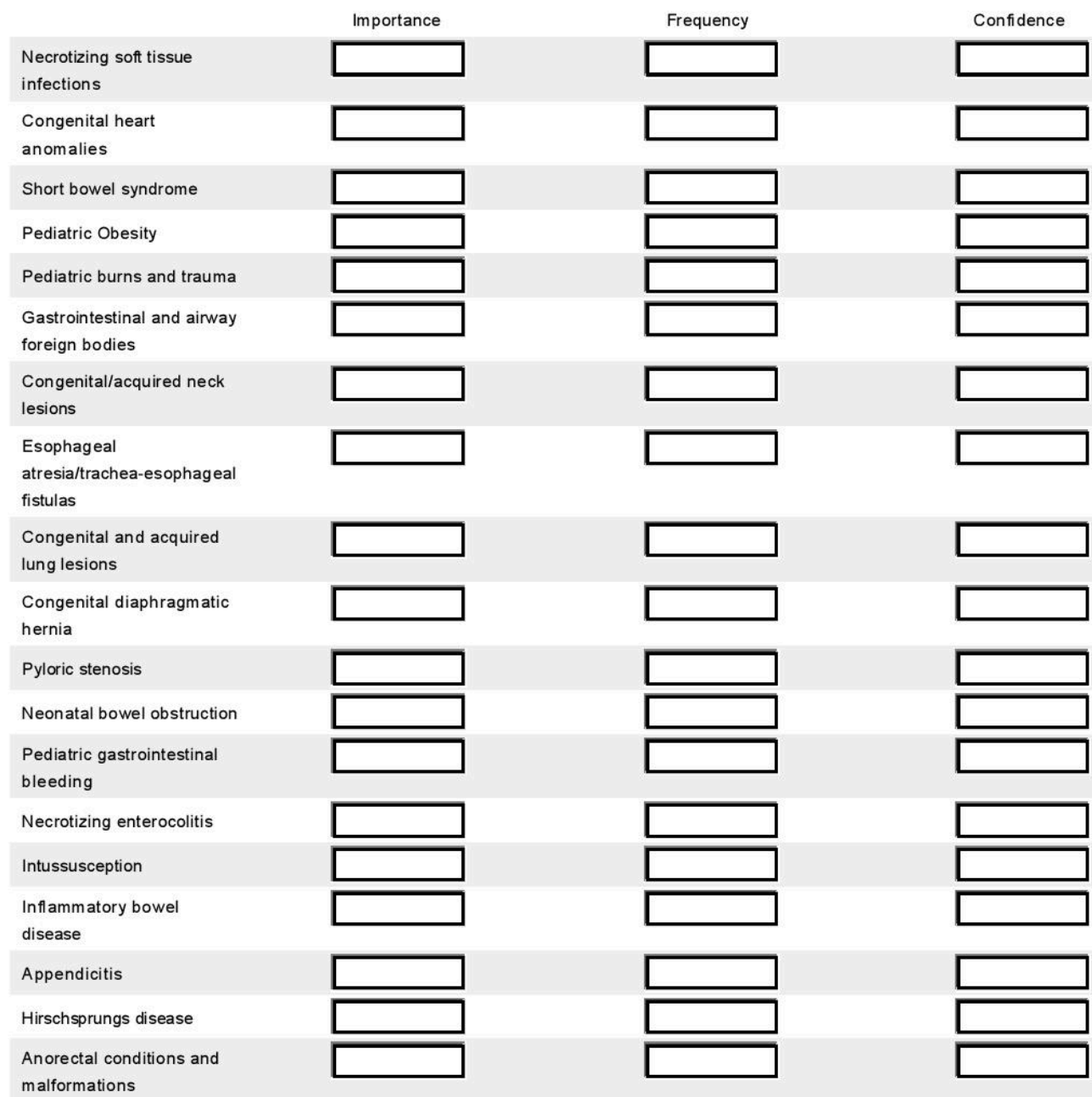




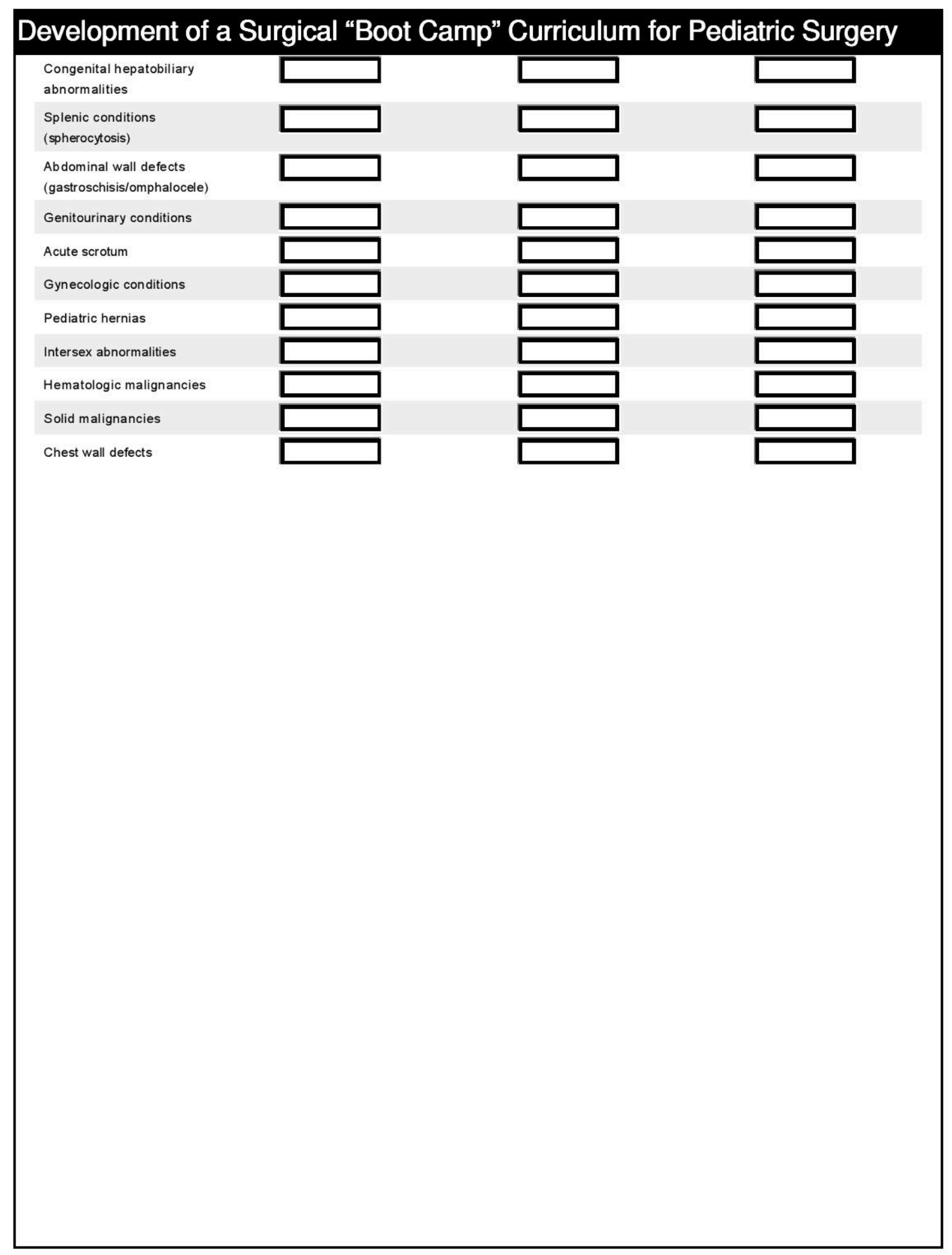




\title{
Development of a Surgical "Boot Camp" Curriculum for Pediatric Surgery
}

\author{
Pediatric and Neonatal Physiology
}

The following page contains a list of PEDIATRIC AND NEONATAL PHYSIOLOGY topics derived from the objectives in training for Pediatric Surgery created by the Royal College of Physicians and Surgeons of Canada. These objectives closely mirror the objectives set forth by the American Council for Graduate Medical Education.

2. Please rate the following topics relating to PEDIATRIC AND NEONATAL PHYSIOLOGY based on the following criteria:

1) The level of IMPORTANCE to new pediatric surgery fellows during their initial transiition to fellowship

2) The FREQUENCY that they are encountered by pediatric surgeons or fellows

3) Your own level of CONFIDENCE in dealing with the topic

Importance

Gastrointestinal physiology

Thermoregulation

Fluid and electrolyte

management

Transfusion therapy and

coagulation

Shock

Pulmonary physiology

Cardiac physiology

PALS

Nutrition

Extra-corporeal life support

Pediatric pain

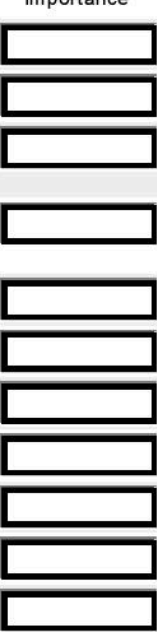

Frequency

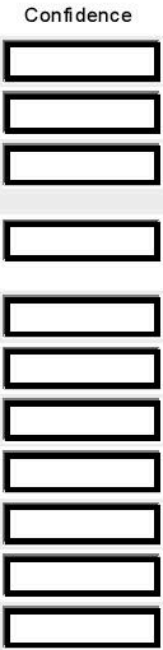




\section{Development of a Surgical "Boot Camp" Curriculum for Pediatric Surgery}

\section{Section 2: Pediatric Medical and Surgical Skills}

The following page contains a list of MEDICALSURGICAL SKILLS derived from the objectives in training for Pediatric Surgery created by the Royal College of Physicians and Surgeons of Canada. These objectives closely mirror the objectives set forth by the American Council for Graduate Medical Education.

3. Please rate each of the following PEDIATRIC MEDICAL/SURGICAL SKILLS based on the following criteria: 1) The level of IMPORTANCE to new pediatric surgery fellows during their initial transiition to fellowship

2) The FREQUENCY that they are applied by pediatric surgeons or fellows

3) Your own level of CONFIDENCE in applying the skill

Vascular access
Endotracheal intubation
Chest tube insertion
Dialysis catheter insertion
Tracheostomy
ECLS cannulation
Advanced Trauma Life
Support
Pediatric Advanced Life
Support
Intraosseous needle
insertion
Clinical and Radiologic
clearance of C-spine
Fasciotomy
Upper Endoscopy
Sigmoidoscopy
Advanced Laparoscopy
Advanced Thoracoscopy
Bronchoscopy
Emergency department
thoracotomy
Trauma Laparotomy
Ultrasound guided
procedures
FAST




\section{Development of a Surgical "Boot Camp" Curriculum for Pediatric Surgery}

Section 3: Format for a Pediatric Surgery Boot Camp

The following questions include multiple choice format and open-ended questions designed to be answered in short answer or single word format.

4. What is an appropriate duration for a pediatric surgery Boot Camp designed to prepare new fellows with the essential skills needed at the start of their fellowship?
1-2 days
3-4 days
5-6 days
7 days or more
other (please specify)

5. Which methods would be best suited for teaching MEDICAL/SURGICAL DIAGNOSES at a pediatric surgery Boot Camp? Please select all that apply:
$\square$ a. Didactic lectures
$\square$ b. Problem/Case based learning
$\square$ c. Interactive small group learning
$\square$ d. E-learning (online tutorials)
$\square$ e. Standardized patients
$\square$ f. Low tech simulation (task trainers)
$\square$ g. High tech simulation (SimMan 3G)
$\square$ h. Telephone simulation (mock ward/clinical pages)
$\square$ i. Animal labs 


\section{Development of a Surgical "Boot Camp" Curriculum for Pediatric Surgery}

6. Which methods would be best suited for teaching PEDIATRIC AND NEONATAL PHYSIOLOGY at a pediatric surgery Boot Camp? Please select all that apply:

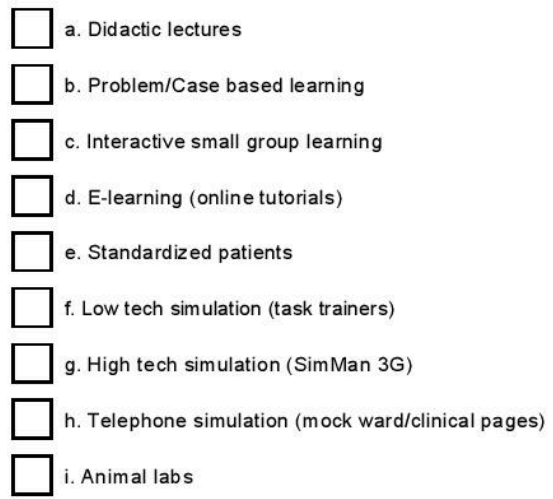

7. Which teaching methods would be best suited for teaching MEDICAL/SURGICAL SKILLS topics at a pediatric surgery Boot Camp? Please select all that apply:

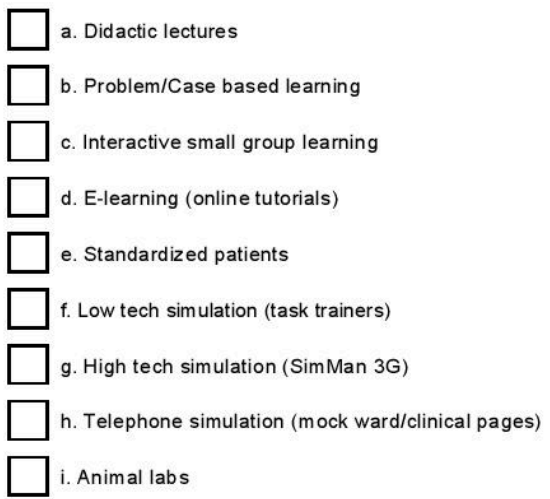

8. What should the main focus of a pediatric surgery boot camp be? (Please rank in order of importance, with 1 being most important and 3 being least important)

Medical and surgical DIAGNOSES

Pediatric and neonatal PHYSIOLOGY

Medical and surgical SKILLS

9. In addition to medical/surgical knowledge and skills competencies, what additional competencies do you feel should be addressed through a pediatric surgery Boot Camp curriculum? 


\section{Development of a Surgical "Boot Camp" Curriculum for Pediatric Surgery}

10. What level of training are you?

First year fellow

second year fellow

Staff

Other

11. What is your gender?

Male

Female

12. In which country did you complete your general surgery residency training?

Canada

Ousa

Other

13. If you have any additional comments, please feel free to include them below. 
APPENDIX D: Example of Likert Scale Used in Needs Assessment Survey

\begin{tabular}{|c|c|c|}
\hline Likert Value & Importance & Frequency \\
\hline 1 & Not at all important & Less than once per year \\
\hline $\mathbf{2}$ & Somewhat important & Once every 6 months-1 year \\
\hline $\mathbf{3}$ & Moderately important & Once every 1-6 months \\
\hline $\mathbf{4}$ & Important & Once every 1-4 weeks \\
\hline 5 & Very important & More than once per week \\
\hline
\end{tabular}




\section{APPENDIX E: Survey Consent Form}

TITLE: Boot Camp Training for Pediatric General Surgery Fellows: Is there a Need for it to Ease the Transition to Fellowship?

SPONSOR: Department of Surgery, Calgary, Alberta Health Services

INVESTIGATORS: PRINCIPAL INVESTIGATOR:

DR. ELIZABETH ODDONE-PAOLUCCI

CO-INVESTIGATORS: $\quad$ DR. STEVEN LOPUSHINSKY

DR. JOCELYN LOCKYER

Before agreeing to participate in this research study, it is important that you read and understand the following explanation of the proposed study. This consent form describes the procedures, benefits, risks and discomforts of the study. It also describes your right to withdraw from the study at any time. This consent form is only part of the process of informed consent. It should give you the basic idea of what the research is about and what your participation will involve. If you would like more details about what is mentioned here or information that is not included here, please ask. Take the time to read this carefully and to understand any accompanying information.

\section{BACKGROUND}

Pediatric surgery is unique amongst surgical and medical specialties in that trainees obtain their initial specialization in adult surgery before embarking upon the world of pediatrics. In North America, this involves five years of adult general surgery training followed by two years of pediatric surgery subspecialty training. At the start of subspecialty training, individuals may have varying degrees of exposure to pediatric surgery and varying comfort levels in addressing urgent and non-urgent pediatric surgical and medical issues. Furthermore, the transition from resident to fellow comes with the sense of increased personal responsibility towards patients. Not surprisingly, during informal discussions with both new fellows and pediatric surgery staff, the initial transition into the fellowship was described as a rather stressful experience. Given this common sentiment between current fellows and staff, curricula needs to be established to help ease the transition into the pediatric surgery subspecialty training.

\section{WHAT IS THE PURPOSE OF THE STUDY?}

The main objective of this study is to apply Kern's model to the development of a pediatric surgery "Boot Camp" curriculum, designed to help with the transition into pediatric surgery training. In this first part of the study, we will be performing a targeted needs assessment to identify the key components of such a "Boot Camp" curriculum from the perspective of the pediatric surgery fellow and other key stakeholders.

\section{WHAT WOULD I HAVE TO DO?}

- As a participant in the study, you will be invited to participate in an online, anonymous survey.

- You will be asked to fill out questions regarding various medical expert CanMEDS competencies and other questions regarding curricular content and design for a pediatric surgery boot camp.

- The survey should take about 10-20 minutes to fill out. 


\section{WHAT ARE THE RISKS?}

There are no anticipated physical, psychological, social, or economic risks posed to participants.

\section{WILL I BENEFIT IF I TAKE PART?}

If you agree to participate in this study there may or may not be a direct benefit to you. By participating in this study, you will get an opportunity to provide information regarding areas you feel are most important to cover when designing a pediatric general surgery Boot Camp curriculum. This is your chance to provide the research and decision-making community with important feedback on this topic. Thus, your responses are highly valued and could provide the foundation of such a Boot Camp, benefiting future generations of pediatric surgery fellows and possibly improving patient care during the initial transition into fellowship.

\section{DO I HAVE TO PARTICIPATE?}

Voluntariness and Withdrawal of consent

Your participation in this study is strictly voluntary and you may withdraw from the study at any time without consequences. No one will know who has or has not participated in the study. If you choose to participate in the questionnaire assessments, you may end your participation at any time. Completion of the electronic survey will be taken as implied consent for your participation in our study.

\section{WILL I BE PAID FOR PARTICIPATING, OR DO I HAVE TO PAY FOR ANYTHING?}

You will not incur any cost for participating in this study. Gift cards will be provided as appreciation for participation in our survey. At the end of the survey, if you would like to receive a gift card, please provide your email address and we will send it electronically to the address provided.

\section{WILL MY RECORDS BE KEPT PRIVATE?}

The online survey is being administered by Survey Monkey ${ }^{\complement}$, an American Software Company. As such, your responses are subject to U.S. laws, including the USA Patriot Act. The risks associated with participation are minimal, however, and similar to those associated with many e-mail programs, such as Hotmail@ and social utilities spaces, such as Facebook@.

Adequate safeguards have been put in place to protect your privacy of any personal information obtained from you in this study. We will store your data anonymously (i.e., stripped of any personal identifying information) in a password-protected and encrypted computer. Only the data analyst will have access to your contact information. No individual information will be disclosed to anyone else. Study investigators will only have access to the non-identifiable data, and only aggregate data will be presented. The University of Calgary Conjoint Health Research Ethics Board will have access to the records.

No direct or indirect harm is foreseen for the study subjects regarding confidentiality because there will not be any connection of your identity to your specific contribution to the research. 


\section{CONSENT}

Your participation and completion in our online survey will be taken as implied consent indicating that you have understood to your satisfaction the information regarding your participation in the research project and agreement to participate as a subject. In no way does this waive your legal rights nor release the investigators or involved institutions from their legal and professional responsibilities. You are free to withdraw from the study at any time. If you have further questions concerning matters related to this research, please contact:

\section{Dr. Elizabeth Oddone Paolucci}

or

\section{Dr. Steven Lopushinsky}

"If you have any questions concerning your rights as a possible participant in this research, please contact The Chair, Conjoint Health Research Ethics Board, University of Calgary."

The University of Calgary Conjoint Health Research Ethics Board has approved this research study. A copy of this consent form has been given to you to keep for your records and reference. 


\section{APPENDIX F: Contribution of Authors}

Student: Dr. Christopher Blackmore (C.B)

Supervisor: Dr. Elizabeth Oddone-Paolucci (E.O.P)

Supervisory Committee Members: Dr. Jocelyn Lockyer (J.L)

Dr. Steve Lopushinsky (S.L)

C.B played an integral role in developing the project including background research and literature review. Further project conceptualization involved contributions from C.B, S.L, J.L and E.O.P.

The initial needs assessment survey was primarily developed by C.B with assistance from J.L, S.L and E.O.P. Survey distribution was undertaken by C.B and S.L. Data analysis was performed by C.B and E.O.P.

The thesis manuscript was written by C.B with supervisory input and guidance from E.O.P, S.L and J.L.

The manuscript comprising Chapter 2 has been accepted for publication in the Journal of Pediatric Surgery. Permission has been granted for use of this article as part of this thesis. The publication date will be in May 2015:

Blackmore C, Lopushinsky S, Lockyer J, Oddone Paolucci E. Targeted needs assessment for a transitional "boot camp" curriculum for pediatric surgery residents. J Ped Surg.

The boot camp meta-analysis manuscript referenced in Chapter 1 has been published in the Journal of Graduate Medical Education. Permission has been granted for the use of this article as part of this thesis: 
Blackmore C, Austin J, Lopushinsky S, Donnon T. Effects of Postgraduate Medical Education 'Boot Camps' on Clinical Skills, Knowledge and Confidence: A Meta-Analysis. J Grad Med Ed. 2014;6(4):643-652. 